\title{
The Severity of COVID-19 and Its Determinants: A Systematic Review and Meta-Analysis in China
}

\author{
Ning Zhang ${ }^{1,2,3}$, Tao Xie ${ }^{1,2}$, Wei Ning ${ }^{1,2}$, Rongxin $\mathrm{He}^{1,2}$, Bin Zhu ${ }^{4, *}$ and Ying Mao ${ }^{1,2, *}$ \\ 1 School of Public Policy and Administration, Xi'an Jiaotong University, Xi'an 710049, China; \\ zhangningati@stu.xjtu.edu.cn (N.Z.); xietao2014077049@stu.xjtu.edu.cn (T.X.); \\ ningwei@stu.xjtu.edu.cn (W.N.); herongxin@stu.xjtu.edu.cn (R.H.) \\ 2 Research Center for the Belt and Road Health Policy and Health Technology Assessment, \\ Xi'an Jiaotong University, Xi'an 710049, China \\ 3 Department of Infectious Disease Epidemiology, Centre for Global Infectious Disease Analysis, \\ Imperial College London, London SW7 2AZ, UK \\ 4 School of Public Health and Emergency Management, Southern University of Science and Technology, \\ Shenzhen 518055, China \\ * Correspondence: zhub6@sustech.edu.cn (B.Z.); mao_ying@xjtu.edu.cn (Y.M.); Tel.: +86-135-3040-5020 (B.Z.); \\ +86-130-8899-9125 (Y.M.)
}

check for

updates

Citation: Zhang, N.; Xie, T.; Ning, W.; He, R.; Zhu, B.; Mao, Y. The Severity of COVID-19 and Its Determinants: A Systematic Review and Meta-Analysis in China. Sustainability 2021, 13, 5305. https://doi.org/ $10.3390 /$ su13095305

Academic Editor:

Haywantee Ramkissoon

Received: 12 April 2021

Accepted: 4 May 2021

Published: 10 May 2021

Publisher's Note: MDPI stays neutral with regard to jurisdictional claims in published maps and institutional affiliations.

Copyright: (C) 2021 by the authors Licensee MDPI, Basel, Switzerland. This article is an open access article distributed under the terms and conditions of the Creative Commons Attribution (CC BY) license (https:/ / creativecommons.org/licenses/by/ $4.0 /)$.

\begin{abstract}
To analyse the prevalence of severe and critical COVID-19 cases and its determinants, a systematic review and meta-analysis were conducted using Review Manager. Four English and two Chinese databases were used to identify and explore the relationships between the severity of COVID-19 and its determinants, with no restrictions on publication date. The odds ratio and $95 \% \mathrm{CI}$ were combined to assess the influencing level of all factors. Twenty-three articles containing a total of 15,828 cases of COVID-19 were included in this systematic review. The prevalence of severe and critical COVID-19 cases was $17.84 \%$ and $4.9 \%$, respectively. A total of 148 factors were identified, which included behavioural, symptom, comorbidity, laboratory, radiographic, exposure, and other factors. Among them, 35 factors could be included in the meta-analysis. Specifically, for example, the male (OR 1.55, 95\% CI 1.42-1.69) and elderly (OR $1.06,95 \%$ CI 1.03-1.10) populations tended to experience severe and critical illness. Patients with cough, dyspnea, fatigue, fever, and gastrointestinal symptoms could have severe and critical diseases. Regarding laboratory results, albumin, aspartate aminotransferase, creatinine, D-dimer, fibrinogen, neutrophils, procalcitonin, platelets, and respiratory rate were potential factors that could be used to predict the severity of COVID.
\end{abstract}

Keywords: systematic review; COVID-19; severity

\section{Introduction}

Coronavirus disease 2019 (COVID-19), initially found in Wuhan, China in December 2019 [1] rapidly developed into a global pandemic. The disease is caused by severe acute respiratory syndrome coronavirus 2 (SARS-CoV-2), with symptoms such as cough, fever, dyspnea, fatigue and other respiratory-tract symptoms. According to COVID-19 data issued by The Johns Hopkins Coronavirus Resource Center (CRC), as of 26 January 2021, there were 99,655.985 confirmed infections and 2138.251 deaths [2]. In China, there were 100,291 confirmed cases and 4814 deaths according to data issued by the National Health Commission of the PRC.

An emergent public-health problem, especially under the circumstance of a completely novel pathogen, is to predict the severity of the disease for each patient. For medical treatment, this issue concerns the reputation of the clinician, the life of the patients, and the therapeutic effects, especially under the conditions of a novel disease and a lack of basic medical resources [3]. 
Disease prevention is related to crucial policy making. Specifically, the severity of disease in different regions could affect the distribution and redeployment of medical staff, equipment, and financial appropriation during the pandemic. In addition, it could need to be traded off against economic-, social-, and personal-freedom costs, such as wearing masks and maintaining social distancing, shutting down factories and schools, and imposing travel restrictions [3-5]. Above all, knowledge about the severity of COVID-19 affects every aspect of the community. According to the guidelines of the World Health Organisation (WHO) and the National Health Commission of the PRC [6,7], the severity of COVID-19 can be classified into four types on the basis of patients' symptoms, laboratory results, and imaging findings at admission: mild, moderate, severe, and critical [8].

Considering the severity of COVID-19, it is crucial to predict the progression of the illness on the basis of evaluable determinants. Previous studies explored and analysed the association between risk factors and the severity of COVID-19. The risk factors could be divided into several types: individual factors (such as gender [9], age [10], residential location [11], and occupation [11]), symptom factors (such as cough [12] and fever [13]), comorbidity factors (such as cancer [14], diabetes [15], psychiatric disorders [16], nephritis [17], and obesity [18]), laboratory factors [19-21], radiographic factors [22], exposure factors [18], treatment factors [23], environmental factors [24-27], social factors [28], and regional factors [29]. In the Chinese context, many empirical studies were conducted to discuss this association [12,30,31]. Among these studies, most reported clinical characteristics of confirmed COVID-19 cases on the basis of different regions, collecting individual, epidemiological, clinical, laboratory, computedtomography-imaging, and outcome data. However, there is no systematic review or meta-analysis that summarises all results on the basis of an analytical framework. In addition, there is no comprehensive classification of all determinants. Therefore, on the basis of the above findings, this study aimed to examine the prevalence of severe COVID-19 and its relationship with other risk factors in China by conducting a systematic review and meta-analysis. This meta-analysis could provide a systemic classification of factors of determinants and most comprehensive results of this subject.

\section{Materials and Methods}

\subsection{Literature Search}

On the basis of the Preferred Reporting Items for Systematic Reviews and MetaAnalyses (PRISMA), we conducted a systematic search on 1 January 2021 of four English language databases, namely, PubMed, EMBASE, the Cochrane Library, and the Web of Science, and two Chinese language databases (CNKI and CBM). Studies with different languages and publication dates in these databases were included. The search strategy was based on the following syntax: (\#1) ("China" OR "Chinese" (Title/Abstract)) AND (\#2) ("COVID-19" OR "coronavirus disease 2019" OR "SARS-CoV-2" (Title/Abstract)) AND (\#3) ("severity" OR "progression" OR "critical" (Title/Abstract)) AND (\#4) ("determinants" OR "factors" OR "indicators" (Title/Abstract)). The full search strategy can be found in Table S1. Additionally, references of the retrieved papers were reviewed to identify additional relevant studies.

\subsection{Study Eligibility}

As inclusion criteria, each study had to: (1) be an original study conducted in China; (2) be published in Chinese or English; (3) report the relationship between at least one determinant and the severity of COVID-19; and (4) provide sufficient information that we could extract the OR and $95 \% \mathrm{CI}$ of the factors from the study. Studies that met one of the following criteria were excluded: (1) having no original data such as reviews, editorials, or communications; (2) sample source or size was not clear; (3) reported insufficient information to allow for the extraction of the OR and 95\% CI for the factors; (3) only studied specific populations such as students, older people, medical staff, or migrants; (4) only reported the mortality of COVID-19 and its determinants. 
Two investigators conducted eligibility assessment by screening and evaluating the titles, abstracts, and full texts of the studies retrieved by the search strategy according to the above-predetermined selection criteria. Two investigators cross-checked assessments. Disagreements on which studies should be included or excluded in this systematic review were subjected to discussion to achieve a group consensus.

\subsection{Data Extraction}

Referring to the checklist of items to consider in the data collection issued by the Cochrane Handbook for Systematic Reviews of Interventions, the following data were extracted from the included full-text studies: first author's name, publication year, region, sample size, patient admission status, severity rate (\%), and critical rate (\%). One author extracted all necessary data, and another author independently reviewed all information. The inter-rater reliability for title screening between the two authors was $95.55 \%$; for abstract screening, it was $93.12 \%$

\subsection{Quality Assessment}

The Newcastle-Ottawa Quality Assessment Scale [32], recommended by the Cochrane Collaboration [33], was applied to assess the quality of the included articles. The standard assessment scale considers patient selection (representativeness of the sample, sample size, nonrespondents and ascertainment of exposure), comparability of the groups (confounding factors are controlled), and outcomes (assessment of outcomes and statistical tests). The highest score is a 7. A score in the range of 5-7 represents a high-quality study, a score in the range of 3-4 means medium-quality research, and a score in the range of 1-2 indicates a poor-quality study. Only medium- and high-quality studies were included in this review. Two reviewers independently evaluated all studies, with disagreements resolved by discussion.

\subsection{Data Synthesis and Statistical Analysis}

In this study, two main outcomes were analysed, namely, severe and critical cases of COVID-19. In addition, the relationship between the severity of COVID-19 and various determinants was researched. Only those rick factors mentioned by at least three articles were included for meta-analysis (for example, gender was discussed by 13 papers, so we included gender in meta-analysis, while exercise was discussed by 2 papers, so it was not meta-analysed). The specific determinant-analysis framework could be formed through previous articles (Figure 1) [13,31,34-36]. Additionally, the reported variable values needed to be the same among selected factors to conduct meta-analysis. The risk-factor selection process is shown in Tables S2 and S3.

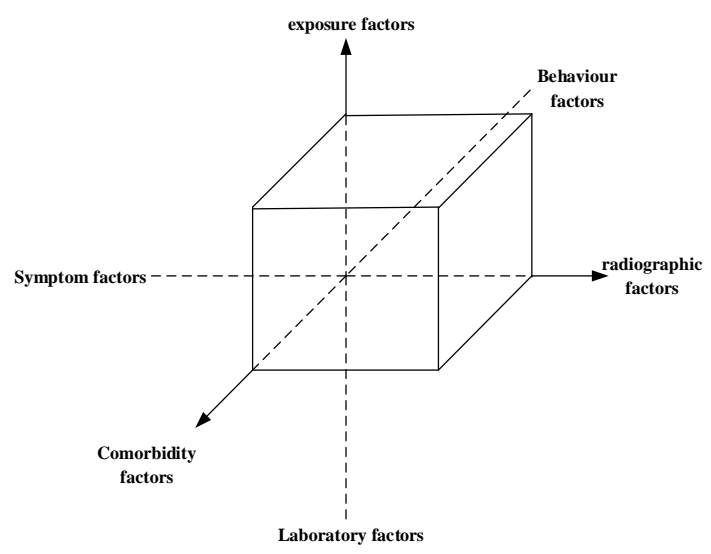

Figure 1. Determinant analysis framework.

In meta-analysis, the odds ratios (ORs) and 95\% CIs were combined to assess the influencing level of all factors. Forest plots were drawn to visualise the data-analysis 
results and to identify the strength of the relationship. The heterogeneity of the pooled results was determined by Cochrane's $Q$ test and Higgins' $I^{2}$ test. Synthesis results with a $p$ value above 1 or an $\mathrm{I}^{2}$ beyond $50 \%$ were considered to be heterogeneous and analysed through a random-effects model. Otherwise, heterogeneity was ignored and analysed with a fixed-effects model.

All statistical analyses were performed by using Stata 13.0 (Stata Corp, College Station, TX, USA) and RevMan 5.3 (The Cochrane Collaboration, Oxford, UK).

\section{Results}

\subsection{Study Characteristics}

A total of 2534 records were identified through our initial systematic search of the literature (PubMed: 435, EMBASE: 699, Cochrane: 5, CNKI: 96, Wang Fang: 363, Web of Science: 936). A total of 1986 unique records were screened on the basis of the title and abstract, and 1798 records were excluded after the removal of duplicate studies, leaving 188 articles for full-text assessment. Among these, nine articles were excluded due to a lack of original data. Seven records were eliminated for unclear samples. A total of 107 articles were removed because they did not report the OR and 95\% CI of the factors of interest. The reviewers ruled out 12 studies because they targeted only a specific population. Thirty articles were excluded because they only studied the mortality of COVID-19 and its determinants. Ultimately, this study included 23 records. In addition, no other studies were included after checking all references of the retrieved articles. The study-selection process is shown in Figure 2.
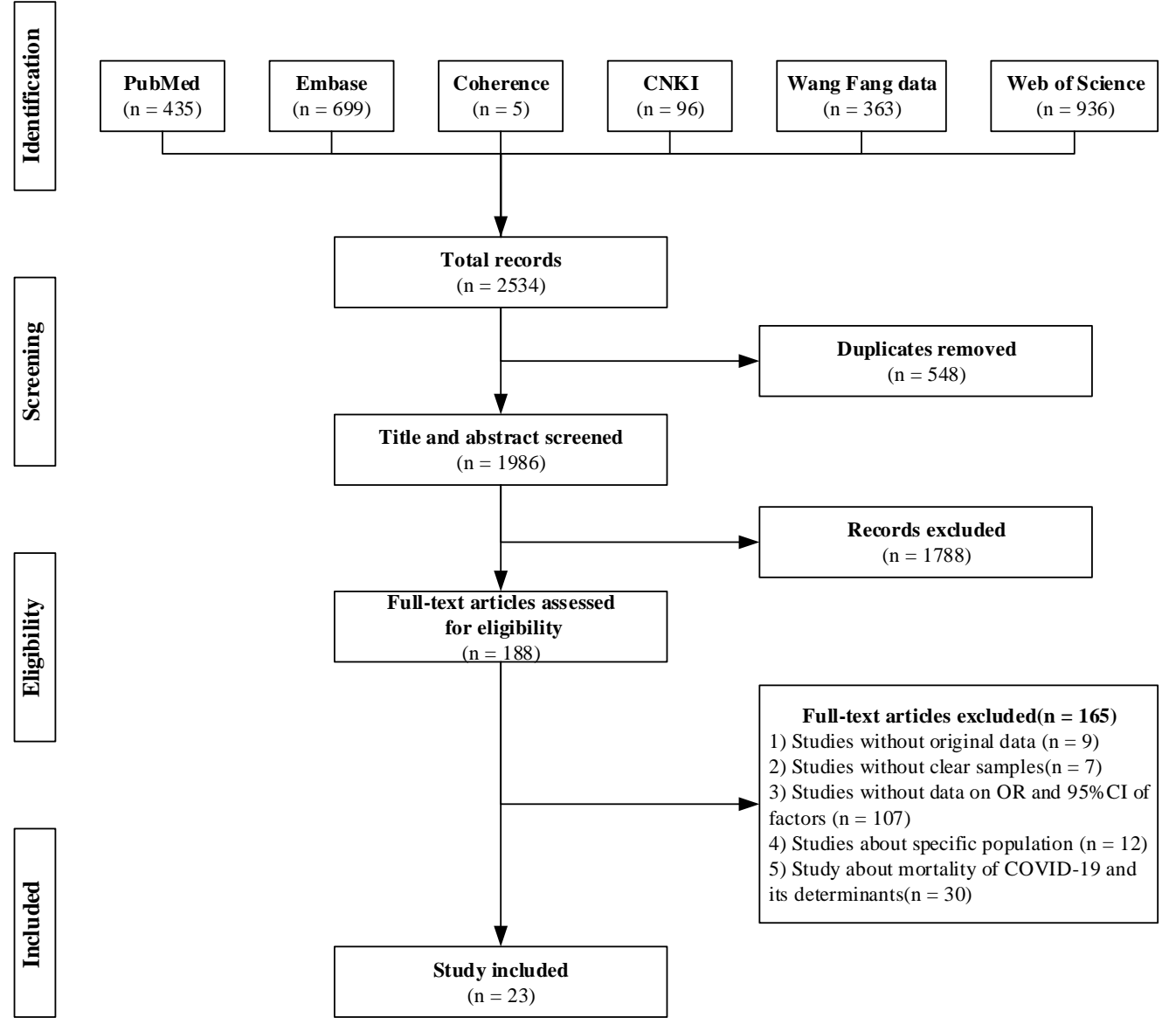

Figure 2. Flow diagram of study selection.

Table 1 presents the main characteristics of all 23 identified studies. These studies were performed in 18 provinces of China in 2020. The included articles contained 
15,828 cases with a median sample size of 333 (range 63-7276). Eleven studies were conducted in Wuhan, Hubei, and 17 in other provinces. In addition, the patient admission time of the included research ranged from 12 December 2019 to 2 April 2020. Twenty-one studies reported the prevalence of severe cases or critical cases, while the two other studies used the progression of COVID-19 as their research object.

Table 1. Characteristics of 23 identified studies.

\begin{tabular}{|c|c|c|c|c|c|c|}
\hline Study & Publication Year & Region & Sample Size & Patient Admission & Severity (\%) & Critical (\%) \\
\hline Liu [37] & November 2020 & Wuhan & 78 & 2019.12.30-2020.01.15 & $11(14.1 \%)$ & - \\
\hline $\mathrm{Xu}[38]$ & July 2020 & Shanghai \Hubei \Anhui & 598 & $2020.01 .01-2020.03 .08$ & $85(14.21)$ & $113(18.90)$ \\
\hline Sun [12] & April 2020 & Beijing & 63 & - & $10(15.9 \%)$ & $9(14.3 \%)$ \\
\hline $\mathrm{Hu}[36]$ & September 2020 & Changsha $\backslash$ Xiangtan & 213 & 2020.01.24-2020.02.16 & \multicolumn{2}{|c|}{$38(17.84 \%)$} \\
\hline Liu [37] & August 2020 & Jiangsu & 625 & 2020.01.10-2020.03.15 & \multicolumn{2}{|c|}{$64(10.2 \%)$} \\
\hline Zhang [31] & July 2020 & Zhejiang & 788 & 2020.01.17-2020.02.12 & $61(7.2 \%)$ & $17(2.2 \%)$ \\
\hline Wang [30] & May 2020 & Tianjin & 131 & Before 2020.02.20 & $22(16.79 \%)$ & - \\
\hline Zhou [38] & September 2020 & Eighteen provinces & 7276 & 2020.01.21-2020.03.02 & - & - \\
\hline Lian [39] & May 2020 & Zhejiang & 465 & 2020.01.17-2020.01-31 & \multicolumn{2}{|c|}{$49(10.54 \%)$} \\
\hline Bi [5] & April 2020 & Shenzhen & 391 & 2020.01.14-2020.02-12 & $35(9 \%)$ & - \\
\hline $\mathrm{Hu}[40]$ & August 2020 & Hunan & 1178 & $2020.01 .16-2020.04 .02$ & $119(11.7 \%)$ & $31(3.0 \%)$ \\
\hline Huang [41] & December 2020 & Henan $\backslash$ Shandong $\backslash$ Hubei & 367 & $2020.02 .10-2020.03 .28$ & $46(12.53 \%)$ & - \\
\hline Cai [42] & July 2020 & Shenzhen & 383 & 2020.01.11-2020.03.26 & $91(23.8 \%)$ & - \\
\hline Zhou [43] & June 2020 & Wuhan & 123 & 2020.01.01-2020.03.31 & $28(22.76 \%)$ & - \\
\hline $\mathrm{Hu}[44]$ & May 2020 & Wuhan & 323 & $2020.01 .08-2020.02 .20$ & $146(45.20 \%)$ & $26(8.05 \%)$ \\
\hline Cen [45] & June 2020 & Wuhan & 1007 & 2020.02.10-2020.03.10 & $222(22.05 \%)$ & $22(2.18 \%)$ \\
\hline $\mathrm{He}[46]$ & August 2020 & Guangzhou & 288 & 2020.01.15-2020.03.10 & $30(10.4 \%)$ & - \\
\hline Wei [47] & August 2020 & Anhui & 167 & Before 2020.03.20 & $30(17.9 \%)$ & - \\
\hline $\operatorname{Li}[48]$ & April 2020 & Wuhan & 548 & 2020.01.26-2020.02.05 & $269(49.1 \%)$ & - \\
\hline Cheng [49] & October 2020 & Jiangxi $\backslash$ Hubei $\backslash$ Guangdong & 252 & 2020.01.19-2020.03.06 & - & $52(20.6 \%)$ \\
\hline Shao [50] & September 2020 & Wuhan $\backslash$ Zhoukou & 126 & $2020.01 .23-2020.03 .23$ & $36(28.67 \%)$ & - \\
\hline Gao [51] & November 2020 & Wuhan & 105 & $2020.02 .10-2020.03 .01$ & - & - \\
\hline Wang [52] & July 2020 & Shenzhen & 333 & 2020.01.10-2020.02.10 & $70(21.0 \%)$ & - \\
\hline
\end{tabular}

A total of 148 factors were extracted from 23 identified studies (Table S3). All were divided into seven categories: 28 behavioural factors, 28 symptom factors, 13 comorbidity factors, 56 laboratory factors, 9 radiographic factors, 7 exposure factors, and 7 other factors. According to the modified Newcastle-Ottawa Scale, the quality scores of all included studies are presented in Table S4. None of the identified studies was excluded due to quality issues.

\subsection{Severe and Critical Cases of COVID-19}

Table 1 shows the percentage of severe and critical cases of COVID-19. On the basis of studies reporting severe cases, 1462 out of $8195(17.84 \%)$ severe cases were found. The highest proportion of severe cases was reported by $\mathrm{Li}(49.1 \%)$, while the lowest was $7.2 \%$. According to research presenting critical cases, the pooled prevalence of critical cases was $4.9 \%(270 / 5512)$. Cheng found the highest percentage of critical cases, $20.6 \%$, whereas $2.18 \%$ was the lowest reported. All studies reported ORs and $95 \%$ CIs of the factors, and examined their relationship with COVID-19. In addition, among all 148 determinants, 35 factors could be included in meta-analysis.

\subsection{Behavioural, Radiographic, and Exposure Factors, and COVID-19 Severity}

Among 28 behavioural factors, sex, age, and smoking history could be included in meta-analysis. In this study, the quantitative synthesis of age and gender were presented by two figures according to the different classification of variables ( $\leq 65$ as reference versus $>65$; younger age as reference versus younger age) (female as reference versus male; male as reference versus female). In addition, no history of smoking was the reference group. Figure 3 shows that the results of related variables apart from gender, with females as a reference $\left(\mathrm{I}^{2}=36 \%, p=0.12\right)$ showing obvious heterogeneity $\left(\mathrm{I}^{2}>50 \%, p<0.1\right)$. Therefore, random-effects analysis was used; age $\leq 65$ as a reference $(Z=4.32, p<0.0001)$, younger age as a reference $(Z=3.97, p<0.0001)$, and gender with female as a reference 
$(Z=9.69, p<0.00001)$ were associated with the severity of COVID-19. Specifically, the population aged $>65$ (OR 3.06, 95\% CI 1.84-5.09), the elderly population (OR 1.06, 95\% CI 1.03-1.10), and men (OR 1.55, 95\% CI 1.42-1.69) showed a higher probability of having severe disease than that of the younger generation and women. In addition, the $p$ value of sex with the male group as the reference and a history of smoking were not significant.

Age ( $\leq 65$ reference)

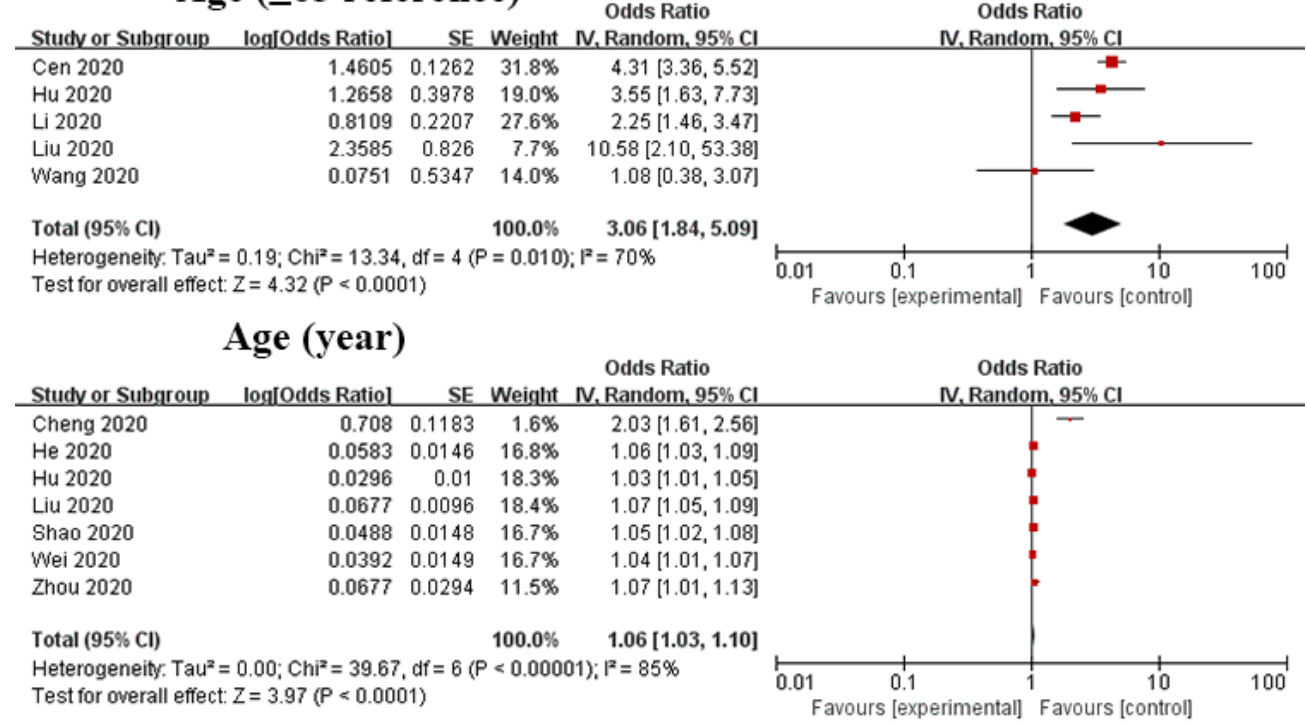

Gender (female reference)

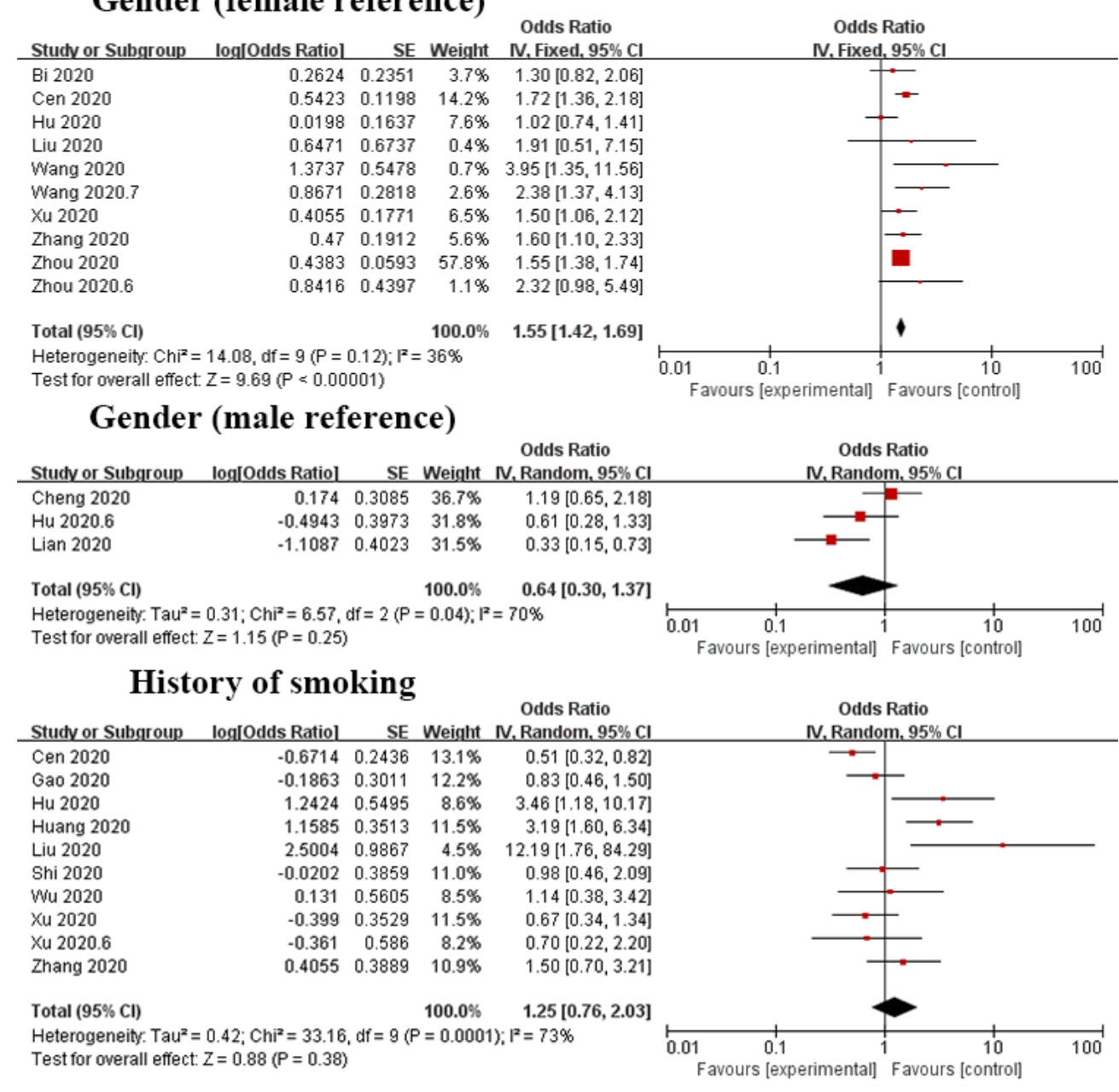

Figure 3. Forest plot of behavioural, radiographic, and exposure factors. 
According to Figure 3, among all of these factors, only bilateral lung involvement and exposure history could be included in meta-analysis. Bilateral lung involvement $\left(\mathrm{I}^{2}=10 \%\right.$, $p=0.34$ ) was conducted through fixed-effects analysis, and bilateral lung involvement in COVID-19 indicates a more severe disease (OR 1.78, 95\% CI 1.29-2.46), while there was no significant relationship between exposure history and the severity of COVID-19.

\subsection{Symptom Factors and Severity of COVID-19}

Cough, dyspnoea, fatigue, fever, headache, muscle or joint pain, sore throat, and gastrointestinal symptoms could be extracted to conduct meta-analysis of all 28 symptom factors. No particular symptom was the reference group of all variables. As shown in Figure 4 , all results of the symptom variables except dyspnoea $\left(\mathrm{I}^{2}=0 \%, p=0.56\right)$, sore throat $\left(\mathrm{I}^{2}=44 \%, p=0.15\right)$, and headache $\left(\mathrm{I}^{2}=46 \%, p=0.14\right)$ had large heterogeneity. Above all, cough $(Z=2.22, p=0.03)$, dyspnoea $(Z=10.22, p<0.00001)$, fatigue $(Z=2.31$, $p=0.02)$, fever $(Z=2.31, p=0.02)$, and gastrointestinal symptoms $(Z=2.95, p=0.003)$ had a significant relationship with the severity of COVID-19, while no significant association was found between headache $(Z=0.54, p=0.59)$, muscle or joint pain $(Z=1.11, p=0.27)$, and sore throat $(Z=0.14, p=0.89)$. In other words, patients with a cough (OR 1.87, 95\% CI 1.08-3.26), dyspnoea (OR 2.86, 95\% CI 2.34-3.50), fatigue (OR 2.86, 95\% CI 1.08-2.52), fever (OR 1.94, 95\% CI 1.11-3.41), and gastrointestinal symptoms (OR 2.33, 95\% CI 1.33-4.07) tended to suffer from more serious disease.

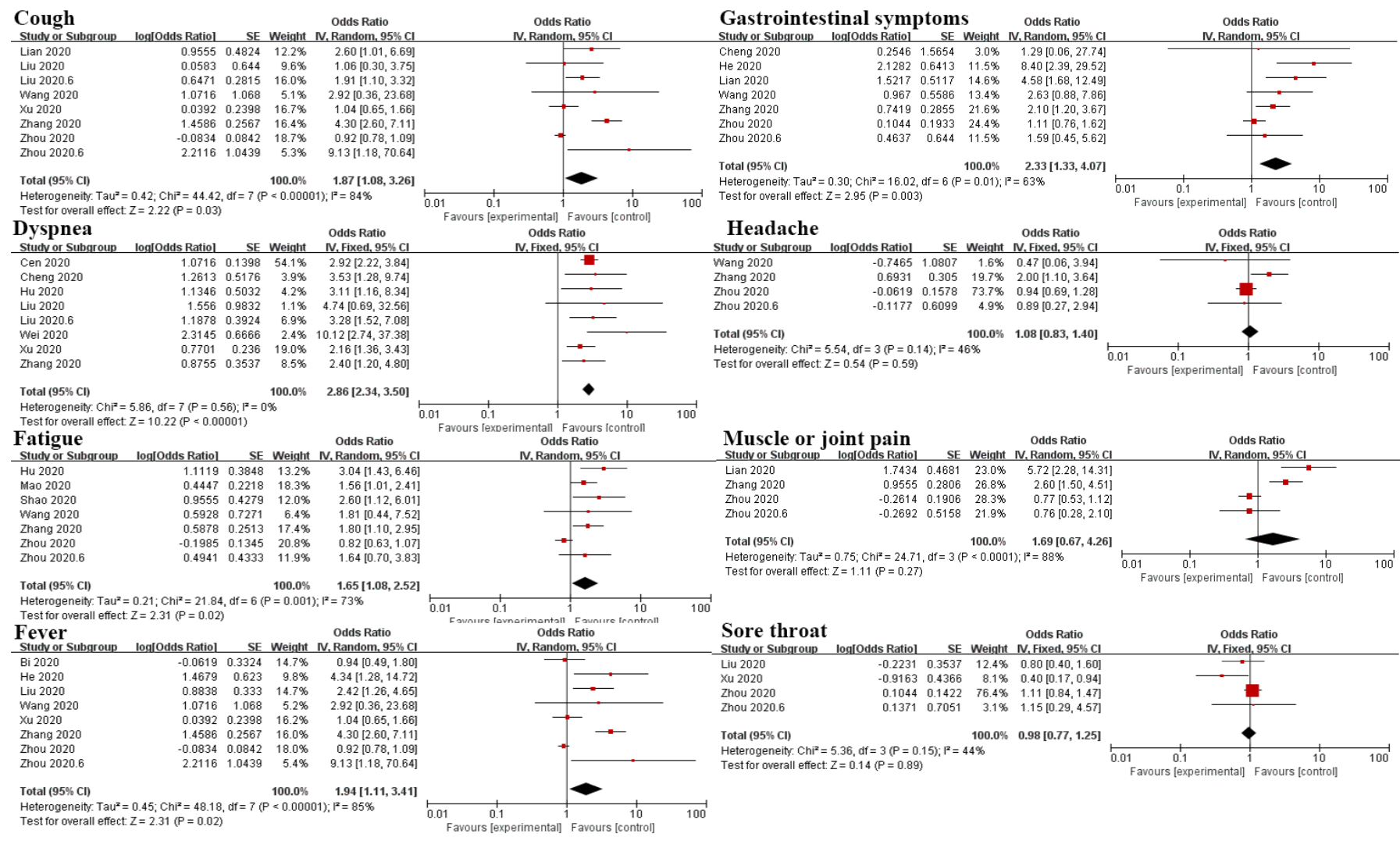

Figure 4. Forest plot of symptom factors.

\subsection{Comorbidity Factors and COVID-19 Severity}

Cancer, cardiovascular diseases, chronic obstructive pulmonary disorder (COPD), diabetes, hypertension, and any other coexisting diseases were identified to conduct meta-analysis. No comorbidity was the reference of all variables. According to Figure 5, only COPD presented distinct heterogeneity, with a $66 \% \mathrm{I}^{2}$ value and a 0.01 $p$ value. Thus, random-effects analysis was adopted. Interestingly, all comorbidities 
were significantly associated with the severity of COVID-19, namely, cancer ( $Z=3.42$, $p=0.0006)$, cardiovascular diseases $(Z=4.80, p<0.00001), \operatorname{COPD}(Z=2.13, p=0.03)$, diabetes $(Z=10.22, p<0.00001)$, and hypertension $(Z=16.66, p<0.00001)$. The possibility of patients with comorbidities to have severe COVID-19 was 3.33 times (cancer, $95 \%$ CI 1.67-6.65), 2.07 times (cardiovascular diseases, 95\% CI 1.54-2.79), 2.69 times (COPD, 95\% CI 1.08-6.70), 2.86 times (diabetes, 95\% CI 2.34-3.50), 3.15 times (hypertension, 95\% CI: 2.75-3.60), and 3.73 times (any other coexisting diseases, 95\% CI: 2.83-4.91) higher than that for patients without comorbidities.

\subsection{Laboratory Factors and COVID-19 Severity}

Among all 56 laboratory factors, 16 factors could be analysed by meta-analysis. According to Figure 6, in this study, the results of the influence of albumin $(<40 \mathrm{~g} / \mathrm{L}$ versus $\geq 40 \mathrm{~g} / \mathrm{L}$ as the reference; low value as reference versus high value), D-dimer ( $\leq 1 \mathrm{mg} / \mathrm{ML}$ as reference versus $>1 \mathrm{mg} / \mathrm{ML}$; low value as reference versus high value), lymphocytes $(\leq 1.1 \times 109 / \mathrm{L}$ as reference versus $>1.1 \times 109 / \mathrm{L}$; low value as reference versus high value) and procalcitonin (PCT) $(\leq 0.05 \mu / \mathrm{L}$ as reference versus $>0.05 \mu / \mathrm{L}$; low values as reference versus high value) were divided into two parts on the basis of classification. The reference groups of the other variables were the low-value group. In consideration of heterogeneity, apart from albumin with $\geq 40 \mathrm{~g} / \mathrm{L}$ as a reference $\left(\mathrm{I}^{2}=0 \%, p=0.42\right)$, D-dimer with $\leq 1 \mathrm{mg} / \mathrm{ML}$ as a reference $\left(\mathrm{I}^{2}=0 \%, p=0.44\right)$, fibrinogen $\left(\mathrm{I}^{2}=0 \%, p=0.39\right)$, procalcitonin (PCT) $\left(\mathrm{I}^{2}=0 \%, p=0.39\right)$, and platelets $\left(\mathrm{I}^{2}=0 \%\right.$, $p=0.85)$, all of the other variables were analysed by random effects.

Albumin $\geq 40 \mathrm{~g} / \mathrm{L}$ as a reference $(Z=9.25, p<0.000001)$, albumin $(Z=5.44$, $p<0.000001)$, aspartate aminotransferase (AST) $(Z=2.09, p=0.04)$, creatinine $(Z=1.95$, $p=0.05)$, d-dimer with $\leq 1 \mathrm{mg} / \mathrm{ML}$ as a reference $(\mathrm{Z}=7.73, p<0.00001)$, fibrinogen $(Z=6.85, p<0.00001)$, neutrophils $(Z=2.07, p=0.04)$, procalcitonin (PCT) with $\leq 0.05 \mu / L$ as a reference $(Z=5.67, p<0.00001)$, platelets $(Z=3.66, p=0.0003)$ and respiratory rate $(Z=2.53, p=0.01)$ had a significant relationship with the severity of COVID-19. Apart from lymphocytes and platelet counts, the higher the other factors were, the more severe a patient's condition was. 


\begin{tabular}{|c|c|c|c|c|c|c|c|}
\hline \multicolumn{4}{|c|}{ Any other coexisting diseases } & Odds Ratio & \multirow{2}{*}{\multicolumn{3}{|c|}{$\begin{array}{l}\text { Odds Ratio } \\
\text { IV. Fixed, 95\% C }\end{array}$}} \\
\hline Studv or Subgroup & log[Odds Ratio] & & Weight & IV. Fixed, 95\% Cl & & & \\
\hline Zhang 2020 & 1.335 & 0.2136 & $43.3 \%$ & $3.80[2.50,5.78]$ & & $=-$ & \\
\hline Wei 2020 & 1.1378 & 0.4199 & $11.2 \%$ & $3.12[1.37,7.10]$ & & & \\
\hline Wang 2020 & 0.7467 & 0.4885 & $8.3 \%$ & $2.11[0.81,5.50]$ & & & \\
\hline Shao 2020 & 1.2892 & 0.418 & $11.3 \%$ & $3.63[1.60,8.24]$ & & & \\
\hline Lian 2020 & 1.1217 & 0.3827 & $13.5 \%$ & $3.07[1.45,6.50]$ & & $\longrightarrow$ & \\
\hline Huang 2020 & 2.0215 & 0.3981 & $12.5 \%$ & $7.55[3.46,16.47]$ & & $\longrightarrow$ & \\
\hline Total $(95 \% \mathrm{Cl})$ & & & $100.0 \%$ & $3.73[2.83,4.91]$ & & & \\
\hline \multirow{2}{*}{\multicolumn{4}{|c|}{$\begin{array}{l}\text { Heterogeneity. } \mathrm{Chi}^{2}=4.95, \mathrm{df}=5(P=0.42) ; 1^{2}=0 \% \\
\text { Test for overall effect: } Z=9.36(P<0.00001)\end{array}$}} & & $\stackrel{\circ}{0.01}$ & 10 & 100 \\
\hline & & & & & Favours lexperimentall & Favours [controll & \\
\hline \multicolumn{4}{|c|}{ Cancer } & Odds Ratio & Odds & Ratio & \\
\hline \multicolumn{4}{|c|}{$\begin{array}{rrrr}\text { Studv or Subgroup } & \text { loglOdds Ratiol } & \text { SE } & \text { Weight } \\
\text { Cheng 2020 } & 24998 & 10618 & 110 \%\end{array}$} & IV. Fixed, $95 \% \mathrm{Cl}$ & IV. Fixed. & $1,95 \% \mathrm{Cl}$ & \\
\hline Cheng 2020 & 2.4998 & 1.0618 & $11.0 \%$ & $12.18[1.52,97.60]$ & & & \\
\hline Xu 2020 & 0.7875 & 0.4211 & $70.0 \%$ & $2.20[0.96,5.02]$ & & & \\
\hline Zhang 2020 & 1.9879 & 0.8074 & $19.0 \%$ & $7.30[1.50,35.53]$ & & & \\
\hline \multirow{2}{*}{\multicolumn{4}{|c|}{$\begin{array}{l}\text { Heterogeneity: } \mathrm{Ch}^{2}=3.41, \mathrm{df}=2(P=0.18) ; \mathrm{I}^{2}=41 \% \\
\text { Test for overall effect: } Z=3.42(P=0.0006)\end{array}$}} & $3.33[1.67,6.65]$ & & & \\
\hline & & & & & ${ }^{0.01} \stackrel{0.1}{0.1}{ }^{1}$ & $\begin{array}{c}10 \\
\text { Favours [control] }\end{array}$ & 100 \\
\hline \multicolumn{3}{|c|}{ Cardiovascular disease } & & Odds Ratio & Odds $\mathrm{F}$ & Ratio & \\
\hline Studv or Subgroup & log[Odds Ratiol & SE & Weight & IV. Fixed, $95 \% \mathrm{Cl}$ & N. Fixed, & $1,95 \% \mathrm{Cl}$ & \\
\hline Cen 2020 & 0.8796 & 0.2742 & $30.7 \%$ & $2.41[1.41,4.12]$ & & & \\
\hline Cheng 2020 & 1.8547 & 0.6579 & $5.3 \%$ & $6.39[1.76,23.20]$ & & & \\
\hline Dong 2020 & 0.9087 & 0.3968 & $14.7 \%$ & $2.48[1.14,5.40]$ & & & \\
\hline He 2020 & 0.9881 & 0.3911 & $15.1 \%$ & $2.69[1.25,5.78]$ & & & \\
\hline Xu 2020 & 0.1169 & 0.3429 & $19.6 \%$ & $1.12[0.57,2.20]$ & 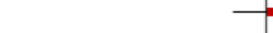 & - & \\
\hline Xu 2020.6 & 0.1476 & 0.4737 & $10.3 \%$ & $1.16[0.46,2.93]$ & & & \\
\hline Zhang 2020 & 0.9163 & 0.7281 & $4.4 \%$ & $2.50[0.60,10.42]$ & & & \\
\hline \multirow{3}{*}{\multicolumn{5}{|c|}{$\begin{array}{l}\text { Heterogeneity. Chi } i^{2}=8.63, \mathrm{df}=6(P=0.20) ;\left.\right|^{2}=30 \% \\
\text { Test for overall effect. } Z=4.80(P<0.00001)\end{array}$}} & & $\diamond$ & \\
\hline & & & & & $0.01 \quad 0.1$ & 10 & 100 \\
\hline \multirow{2}{*}{\multicolumn{3}{|c|}{$\begin{array}{l}\text { Test for overall effect } Z=4.80(P<0.00001) \\
\text { COPD }\end{array}$}} & & & Favours lexperimentall & Favours Icontroll & \\
\hline & & & & Odds Ratio & $\begin{array}{r}\text { Odds } \\
\end{array}$ & Ratio & \\
\hline Studv or Subgroup & loglOdds Ratio] & SE & Weight & N, Random, $95 \% \mathrm{Cl}$ & IV, Rando & $\mathrm{m}, 95 \% \mathrm{Cl}$ & \\
\hline Cen 2020 & 1.2759 & 0.188 & $27.5 \%$ & $3.58[2.48,5.18]$ & & $\rightarrow-$ & \\
\hline Cheng 2020 & 0.2546 & 0.9772 & $12.7 \%$ & $1.29[0.19,8.76]$ & & & \\
\hline Gao 2020 & -0.4292 & 0.7523 & $16.4 \%$ & $0.65[0.15,2.84]$ & & 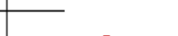 & \\
\hline Hu 2020 & 1.8399 & 0.9352 & $13.3 \%$ & $6.30[1.01,39.36]$ & & & \\
\hline Xu 2020 & 0.1017 & 0.6222 & $19.0 \%$ & $1.11[0.33,3.75]$ & & & \\
\hline Zhang 2020 & 3.7377 & 1.0962 & $11.1 \%$ & $42.00[4.90,360.04]$ & & & $\longrightarrow$ \\
\hline Total $(95 \% \mathrm{Cl})$ & & & $100.0 \%$ & $2.69[1.08,6.70]$ & & & \\
\hline \multicolumn{5}{|c|}{$\begin{array}{l}\text { Heterogeneity: } \mathrm{Tau}^{2}=0.75 ; \mathrm{Chi}^{2}=14.65, \mathrm{df}=5(\mathrm{P}=0.01) ; \mathrm{I}^{2}=66 \% \\
\text { Test for overall effect: } Z=2.13(\mathrm{P}=0.03)\end{array}$} & 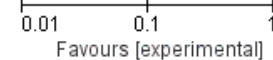 & $\begin{array}{l}10 \\
1 \\
\text { Favours [control] }\end{array}$ & 100 \\
\hline \multicolumn{4}{|c|}{ Diabetes } & Odds Ratio & Odds & Ratio & \\
\hline Study or Subgroup & log[Odds Ratio] & SE & Weight & N. Fixed, $95 \% \mathrm{Cl}$ & IV. Fixed & & \\
\hline Cen 2020 & 1.0716 & 0.1398 & $54.1 \%$ & $2.92[2.22,3.84]$ & & 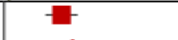 & \\
\hline Cheng 2020 & 1.2613 & 0.5176 & $3.9 \%$ & $3.53[1.28,9.74]$ & & & \\
\hline Hu 2020 & 1.1346 & 0.5032 & $4.2 \%$ & $3.11[1.16,8.34]$ & & & \\
\hline Liu 2020 & 1.556 & 0.9832 & $1.1 \%$ & $4.74[0.69,32.56]$ & & & \\
\hline Liu 2020.6 & 1.1878 & 0.3924 & $6.9 \%$ & $3.28[1.52,7.08]$ & & $\rightarrow$ & \\
\hline Wei 2020 & 2.3145 & 0.6666 & $2.4 \%$ & $10.12[2.74,37.38]$ & & & \\
\hline Xu 2020 & 0.7701 & 0.236 & $19.0 \%$ & $2.16[1.36,3.43]$ & & $\rightarrow-$ & \\
\hline Zhang 2020 & 0.8755 & 0.3537 & $8.5 \%$ & $2.40[1.20,4.80]$ & & & \\
\hline Total $(95 \% \mathrm{Cl})$ & & & $100.0 \%$ & $2.86[2.34,3.50]$ & & $\bullet$ & \\
\hline $\begin{array}{l}\text { Heterogeneity: Chi }= \\
\text { Test for overall effect: }\end{array}$ & 5.86, df $=7(P=0.5$ & $56) ; 1^{2}=09$ & & & $\begin{array}{ll} \\
0.01 & 0.1\end{array}$ & $1 \quad 10$ & 100 \\
\hline Test for overall effect: & $Z=10.22(P<0.000$ & 1001) & & & Favours lexperimenta & Favours [control] & \\
\hline Hypentensio & & & & Odds Ratio & Odds $\mathrm{F}$ & Ratio & \\
\hline Studv or Subgroup & log[Odds Ratio] & SE & Weight & IV. Fixed, $95 \% \mathrm{Cl}$ & N. Fixed, & $1,95 \% \mathrm{Cl}$ & \\
\hline Cen 2020 & 0.9322 & 0.1194 & $33.2 \%$ & $2.54[2.01,3.21]$ & & $=$ & \\
\hline Cheng 2020 & 1.3455 & 0.3537 & $3.8 \%$ & $3.84[1.92,7.68]$ & & & \\
\hline Hu 2020 & 1.0188 & 0.4397 & $2.4 \%$ & $2.77[1.17,6.56]$ & & 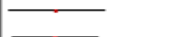 & \\
\hline Hu 2020.6 & 1.008 & 0.3923 & $3.1 \%$ & $2.74[1.27,5.91]$ & & 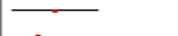 & \\
\hline Li 2020 & 0.6981 & 0.2342 & $8.6 \%$ & $2.01[1.27,3.18]$ & & $\rightarrow$ & \\
\hline Liu 2020 & 0.8154 & 0.8964 & $0.6 \%$ & $2.26[0.39,13.10]$ & & & \\
\hline Liu 2020.6 & 1.0543 & 0.3013 & $5.2 \%$ & $2.87[1.59,5.18]$ & & $\longrightarrow$ & \\
\hline Shao 2020 & 1.1787 & 0.4714 & $2.1 \%$ & $3.25[1.29,8.19]$ & & & \\
\hline Wang 2020 & 1.311 & 0.1584 & $18.9 \%$ & $3.71[2.72,5.06]$ & & $=$ & \\
\hline Хu 2020 & 1.5019 & 0.1808 & $14.5 \%$ & $4.49[3.15,6.40]$ & & $\rightarrow$ & \\
\hline Zhang 2020 & 1.5892 & 0.2503 & $7.6 \%$ & $4.90[3.00,8.00]$ & & & \\
\hline Total $(95 \% \mathrm{Cl})$ & & & $100.0 \%$ & $3.15[2.75,3.60]$ & & $\bullet$ & \\
\hline $\begin{array}{l}\text { Heterogeneity. } \mathrm{Chi}^{2}= \\
\text { Test for overall effect: }\end{array}$ & $\begin{array}{l}15.72, d f=10(P= \\
Z=16.66(P<0.00\end{array}$ & $\begin{array}{l}0.11) ; 1^{2}= \\
0001)\end{array}$ & & & $\begin{array}{lcl} \\
0.01 & 0.1 & 1 \\
\text { Favours [experimental] }\end{array}$ & $\begin{array}{l}10 \\
\text { Favours [control] }\end{array}$ & $100^{\circ}$ \\
\hline
\end{tabular}

Figure 5. Forest plot of comorbidity factors. 


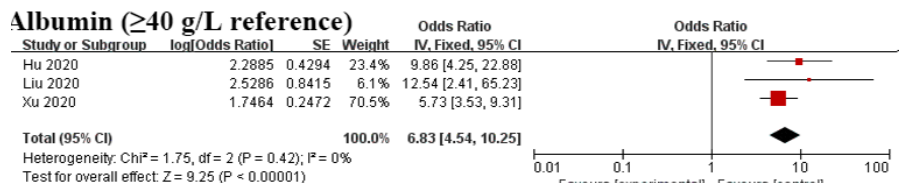

Creatine kinase
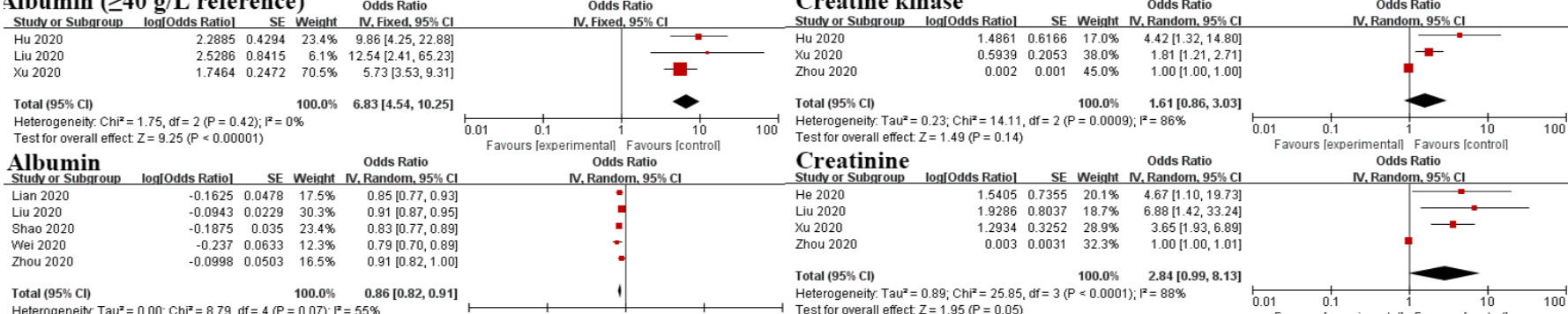

Heterogeneity. Taü $=0.23 ; \mathrm{Chi}^{2}=14.11$, af $=2(P=0.0009) ;$
Test for overall effect $Z=1.49 \%(P=0.14)$

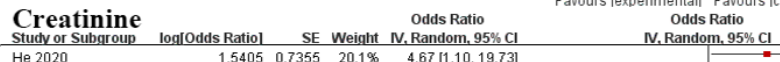
$\begin{array}{lllll}\text { He } 2020 & 1.5405 & 0.7355 & 20.1 \% & 4.67[1.10,19.73] \\ \text { Liu } 2020 & 1.9286 & 0.8037 & 18.7 \% & 6.88[1.42,33.24]\end{array}$ $\begin{array}{lllll}\text { Liu } 2020 & 1.9286 & 0.8037 & 18.7 \% & 6.88[1,42,33.24] \\ \text { Xu } 2020 & 1.2934 & 0.3252 & 28.9 \% & 3.65[1.93,6.89]\end{array}$ Zhou $2020-0.003 \quad 0.003132 .3 \% \quad 1.00[1.00,1.01]$

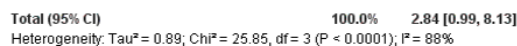
Heterogeneity. Tau ${ }^{2}=0.89 ; C \mathrm{Cl}^{2}=25.85$, df $=3(P<0.0001) ; P^{2}=88 \%$
Test for overall effect $Z=1.95(P=0.05)$ Total $(95 \% \mathrm{Cl})$
Heterogeneity Tau $^{2}=0.00 ; \mathrm{Ch}^{\mathrm{z}}=8.79, \mathrm{df}=4(\mathrm{P}=0.07) ; \mathrm{P}=55 \%$ ALST for overall effect: $Z=5.44(P<0.00001)$

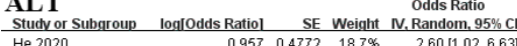
$\begin{array}{lrrrr}\text { He 2020 } & 0.957 & 0.4772 & 18.7 \% & 2.60[1.02,6.63] \\ \text { Hu 2020 } & 1.2208 & 0.4194 & 19.8 \% & 3.39[1.49,7.71] \\ \text { Liu 2020 } & 0.0315 & 0.7637 & 13.6 \% & 1.03[0.23,4.61]\end{array}$

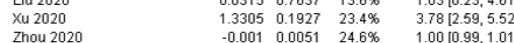
Total $(95 \% \mathrm{Cl}) \quad 100.0 \% \quad 2.09[0.91,4.77$ Heterogeneity. Tau $=0.73 ; C \mathrm{Ch}^{2}=60.21$, df $=4(P<0.00001) ;{ }^{2}=93 \%$
Test for overall effect $Z=1.74(P=0.08)$

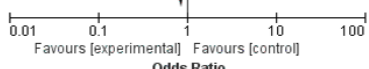

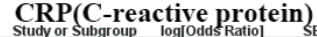

Odds Ratio
SE Weight $\mathrm{N}$. Random, $95 \% \mathrm{Cl}$
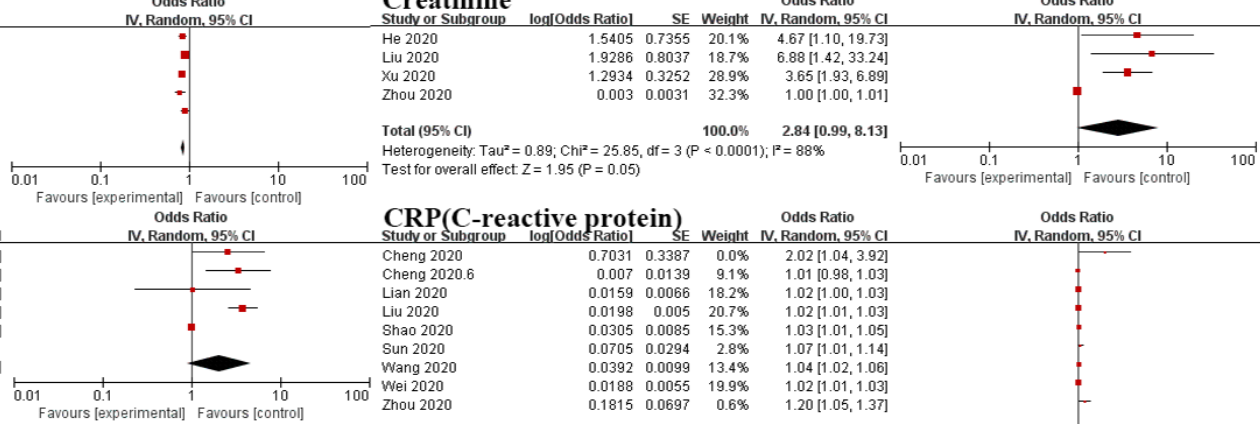

Cheng 2020

Cheng 2020
Lian 2020

Liu 2020

Shao 2020
Sun 2020

Wang 2020

Whoi 2020

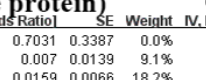

Total $(95 \% \mathrm{Cl})$

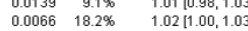

$\left.\begin{array}{llll}0.0198 & 0.005 & 20.7 \% & 1.02[1.01,1.03] \\ 0.0305 & 0.0085 & 15.3 \% & 1.03[1.01,1.05\end{array}\right]$

$\begin{array}{llll}0.0705 & 0.0294 & 2.8 \%\end{array}$

$\begin{array}{llll}0.0392 & 0.0099 & 13.4 \% & 1.04[1.02,1.06\end{array}$

$\begin{array}{rrrr}0.0188 & 0.0055 & 19.9 \% & 1.02[1.01,1.03] \\ 0.1815 & 0.0697 & 0.6 \% & 1.20[105,1.37]\end{array}$

$\begin{array}{rl}100.0 \% & 1.02[1.01,1.04]\end{array}$ Heterogeneity. Tau $=0.00 ; \mathrm{Chi}^{2}=18.57$, df $=8(P=0.02) ; \mathrm{I}^{2}=57$

D-dimer ( $\leq 1$ mg/ML reference) Odds Ratio

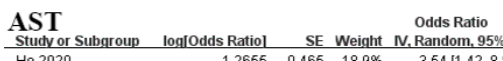

$\begin{array}{rrrrr}\text { He } 2020 & 1.2655 & 0.465 & 18.9 \% & 3.54 \\ \text { Hu } 2020 & 11.42,8.82\end{array}$

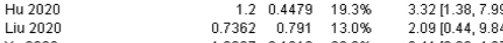

$\begin{array}{lrrrr}\text { Xu 2020 } & 1.2267 & 0.1818 & 23.8 \% & 3.41[2.39,4.87] \\ \text { Zhou 2020 } & 0.005 & 0.0082 & 25.0 \% & 1.01[0.99,1.02]\end{array}$

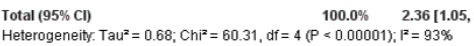

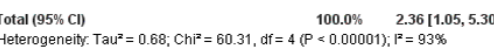

Test for overall effect $Z=2.09(P=0.04)$

\begin{tabular}{lrrrr} 
Studv or Subgroup & loglOdds Ratiol & SE Weight & N. Random, $95 \%$ Cl \\
\hline Hu 2020 & 1.4422 & 0.5678 & $20.7 \%$ & $4.23[1.39,12.87$
\end{tabular} $\begin{array}{lrrrr}\text { Hu } 2020 & 1.4422 & 0.5678 & 20.7 \% & 4.23[1.39,12.81 \\ \text { Xu 2020 } & -0.4308 & 0.3291 & 32.8 \% & 0.65[0.34,1.24 \\ \text { Zhou 2020 } & 0.01 & 0.0432 & 46.5 \% & 1.01[0.93,1.10\end{array}$

$\begin{array}{llll}0.01 & 0.0432 & 46.5 \% & 1.01[0.93,1.10\end{array}$

Total $(95 \% \mathrm{Cl})$

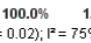
$1.17[0.60,2.31]$ Heterogeneity: $\mathrm{Tau}^{2}=0.25 ; \mathrm{Chi}^{2}=8.16$,
Test for overall effect $Z=0.47(\mathrm{P}=0.64)$

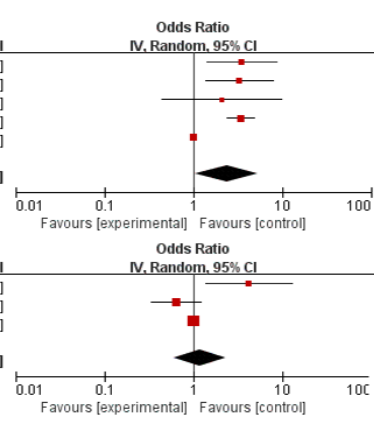

Studvor Subgroup log/odds Ratiol SE Weightit N. Fixed, 95\% CI

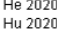

Li 2020

Liu 2020
Xu 2020

$\begin{array}{rrrr}0.6663 & 0.4175 & 9.5 \% & 1.95[0.86,4.41] \\ 0.9895 & 0.3993 & 10.4 \% & 2.69 \\ 0.7723,5.88]\end{array}$

$\begin{array}{llll}0.7793 & 0.2187 & 34.7 \% & 2.18[1.42,3.35]\end{array}$ $\begin{array}{rrrrr}0.5872 & 0.8166 & 2.5 \% & 1.80[0.36,8.91] \\ 1.2664 & 0.1965 & 42.9 \% & 3.55[2.41,5.21]\end{array}$

Total $(95 \% \mathrm{Cl})$ $100.0 \% \quad 2.70[2.10,3.48]$

Heterogeneity $\mathrm{Chi}^{2}=3.75, \mathrm{df}=4(P=0.44) ; \mathrm{F}^{2}=0$
Test for overall effect $Z=7.73(P<0.00001)$

D-dimer

Studv or Subaroup loolOdds Ration SE Weinht Odds Ratio

\begin{tabular}{lrrrr} 
Cheng 2020 & 0.9243 & 0.3416 & $18.3 \%$ & $2.52[1.29,4.92$ \\
Liu 2020 & 0.2469 & 0.0962 & $36.7 \%$ & 1.28 \\
\hline & $11.06,1$
\end{tabular}

$\begin{array}{lllll}\text { Liu } 2020 & 0.2469 & 0.0962 & 36.7 \% & 1.28[1.06,1.55\end{array}$ \begin{tabular}{llrrr} 
Sun 2020 & 1.6702 & 1.4256 & $1.8 \%$ & $5.31[0.33,86.86]$ \\
Wang 2020 & 0.5766 & 0.2841 & $22.0 \%$ & $1.78[1.02,3.11]$ \\
\hline
\end{tabular} Total (95\% CI) $\quad 100.0 \% \quad 1.43[0.97,2.10]$ Heterogeneity: $\operatorname{Tau}^{2}=0.10 ; \mathrm{Ch}^{2}=9.44$, df $=4(P=0.05) ; \mathrm{P}^{2}=58 \%$

PCT (Procalcitonin, $\leq 0.05 \mu / 1$ reference)

Fibrinogen

Studv or Subgroup logiOdds Ratiol SE Weight $\begin{gathered}\text { Odds Ratio } \\ \text { N. Fixed, 95\% C }\end{gathered}$

\begin{tabular}{lrrrr}
\hline Liu 2020 & 0.6259 & 0.1125 & $65.3 \%$ & 1.87 \\
\hline
\end{tabular} Whou 2020

$\begin{array}{llll}1.041 & 0.3458 & 6.9 \% & 2.83[1.44,558\end{array}$

Total (95\% Cl)

$100.0 \% \quad 1.86[1.56,2.23]$

Heterogeneity. Chi $\mathrm{F}^{2}=1.87$, df $=2(P=0.39) ;{ }^{R}=0 \%$
Test for overall effect $Z=6.85(P<0.00001)$

LDH

$\begin{array}{cccc}\text { Study or Subgroup loglodds Ratiol } & \text { SE Weight N. Random. } 95 \% \mathrm{C}\end{array}$

\begin{tabular}{lrrrr}
\hline Hu 2020 & 1.9095 & 0.4489 & $28.8 \%$ & $6.75[2.80,16.27]$ \\
Wei 2020 & 0.007 & 0.0025 & $36.4 \%$ & $1.01[1.00,1.01]$
\end{tabular}

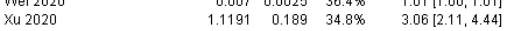

Total (95\% Cl) $100.0 \% \quad 2.57[0.91,7.23]$

Heterogeneity: Tau ${ }^{2}=0.77 ; C h \mathrm{~F}^{2}=52.57, \mathrm{df}=2(P<0.00001) ; \mathrm{F}^{2}=96 \%$
Test for overall effect: $Z=1.78(P=0.07)$

Lymphocytes $(\geq 1.1 \times 109 / \mathrm{L}$ referenceal Ratio

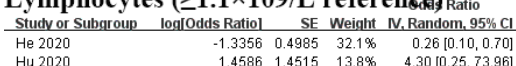

Liu 2020

$\begin{array}{rrrr}-1.3356 & 0.4985 & 32.1 \% & 0.26[0.10,0.70] \\ 1.4586 & 1.4515 & 13.8 \% & 4.30[0.25,73.96]\end{array}$

$\begin{array}{rrrr}0.47 & 1.153 & 18.1 \% & 1.60[0.17,15.33] \\ 0.5988 & 0.3055 & 36.0 \% & 1.82[1.00,3.31]\end{array}$

Total $(95 \% \mathrm{Cl}) \quad 100 \% \quad 1.08[0.29,4.01]$

Heterogeneity. Tau $^{2}=1.16 ; \quad C \mathrm{i}^{2}=11.92$, df $=3(P=0.008) ; \mathrm{F}^{2}=75 \%$
Test for overall effect $Z=0.11(P=0.91)$

Lymphocytes
S Sudver or Subgroup logiOdds Ratiol SE Weight IV. Random. $95 \%$

\begin{tabular}{llrrr} 
& & & \\
Studve or Subgroup & loglodds Ratiol & SE & Weight & N. Random, $95 \%$ Cl \\
\hline Cheng 2020 & -4.6052 & 1.3465 & $0.0 \%$ & $0.01[0.00,0.14]$ \\
Lian 2020 & -1.4439 & 0.5092 & $0.2 \%$ & $0.24[0.09,0.64]$
\end{tabular}

Liu 2020
Shi 2020

Shi 2020
Sun 2020

Wei 2020

$\begin{array}{llll}-1.4439 & 0.5092 & 0.2 \% & 0.24[0.09,0.64] \\ -3.5066 & 0.5605 & 0.2 \% & 0.03[0.01,0.09] \\ 0.6206 & 0.2409 & 1.0 \% & 1.86[1.16,2.98]\end{array}$

$\begin{array}{rrrr}0.6206 & 0.2409 & 1.0 \% & 1.86[1.16,2.98] \\ -0.0111 & 0.0052 & 48.6 \% & 0.99[0.98,1.00]\end{array}$

$\begin{array}{rrrr}-0.0111 & 0.0052 & 48.6 \% & 0.99[0.98,1.00] \\ -0.004 & 0.001 & 49.7 \% & 1.00[0.99,1.00] \\ -1.2588 & 0.5377 & 0.2 \% & 0.28 \\ -2.4769 & 0.6183 & 0.1 \% & 0.08[0.030 .81] \\ -2.03,0.28]\end{array}$

Zhou 2020

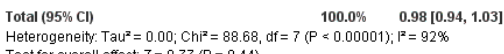

Heterogeneity: Tau" $=0.00 ; \mathrm{Chi}^{2}=88.68$,
Test for overall effect. $Z=0.77(\mathrm{P}=0.44)$

Neutrophils

Studv or Subgroup loglodds Ratiol SE Weight Odds Ratio

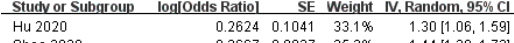

Shao 2020

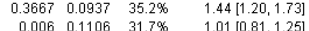

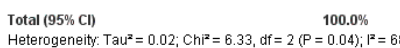
$100.0 \% \quad 1.24[1.01,1.53]$

Test for overall effect. $Z=2.07(P=0.04)$

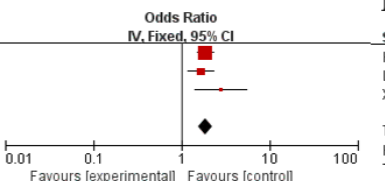

\begin{tabular}{rrrrr} 
Studvor Subqroup & loglOdds Ratiol & SE Weight & $\begin{array}{l}\text { Odds Ratio } \\
\text { N. Fixed.95\% }\end{array}$ \\
\hline He 2020 & 1.6739 & 0.6233 & $34.9 \%$ & $5.33[1.57,18.09$
\end{tabular}

$\begin{array}{rrrrr}\text { He } 2020 & 1.6739 & 0.6223 & 34.9 \% & 5.33[1.157,18.09] \\ \text { Liu } 2020 & 13431 & 0.9894 & 13.9 \% & 3.83[0.55,26.64\end{array}$

$\begin{array}{lllll}\text { Liu } 2020 & 1.3431 & 0.9894 & 13.9 \% & 3.83[0.55,26.64] \\ \text { Xu } 2020 & 2.5713 & 0.5148 & 51.2 \% & 13.08[4.77,35.89\end{array}$

Total $(95 \% \mathrm{Cl})$

$100.0 \% \quad 8.07[3.92,16.60]$

Heterogeneity $C \mathrm{Ch}^{2}=1.89$, df $=2(P=0.39) ; \mathrm{F}^{2}=0 \%$
Test for overall effect. $Z=5.67(P<0.00001)$

PCT (Procalcitonin) Odds Ratio

\begin{tabular}{lrrrr} 
Studv or Subaroup & loglOdds Ratiol & SE Weight & N. Random, 95\% C \\
\hline Cheng 2020 & 1.4816 & 0.4646 & $44.4 \%$ & $4.40[1.77,10.94]$
\end{tabular}

$\begin{array}{llrrr}\text { Cheng } 2020 & 1.4816 & 0.4646 & 44.4 \% & 4.40[1.77,10.94] \\ \text { Shao } 2020 & 4.8135 & 2.1167 & 12.8 \% & 123.16[1.94,7802.16]\end{array}$

Wang $2020 \quad-0.1054 \quad 0.5276 \quad 42.8 \% \quad 0.90[0.32,2.53]$

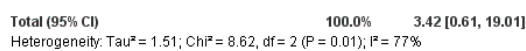

Heterogeneity: Tau ${ }^{2}=1.51 ; C \mathrm{Ch}^{2}=8.62$, df $=2(P=0.01) ; \mathrm{l}^{2}=77 \%$
Test for overall effect. $Z=1.40(P=0.16)$

Platelets

Studv or Subrroup loglodds Ratiol SE Weight N. Fixed, $95 \% \mathrm{CI}$

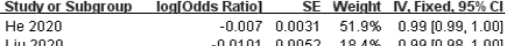

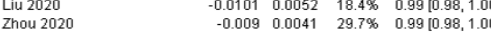

Tol

$\begin{array}{llll}-0.009 & 0.0041 & 29.7 \% & 0.99[0.98,1.00\end{array}$

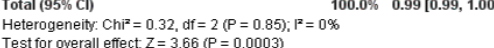

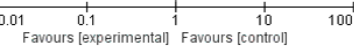

Respiratory rate Odds Ratio

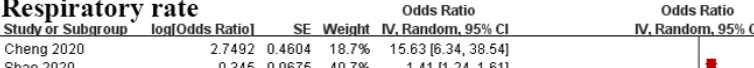

$\begin{array}{lrrrr}\text { Shao 2020 } & 0.345 & 0.0675 & 40.7 \% & 1.41[1.24,1.61] \\ \text { Wang 2020 } & 0.0583 & 0.0723 & 40.6 \% & 1.06[0.92,1.22]\end{array}$

Total $(95 \% \mathrm{Cl}) \quad 100.0 \% \quad 1.97[1.17,3.34]$

Heterogeneity: Tau ${ }^{2}=0.17 ; \mathrm{Ch}^{2}=38.44$, df $=2(P<0.00001) ; \mathrm{F}^{2}=95 \%$

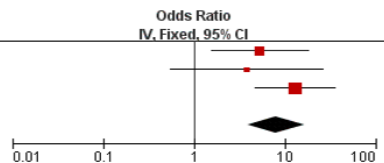

Favours lexperimentall Favours Ico
Odds Ratio
N. Random. $95 \% \mathrm{Cl}$
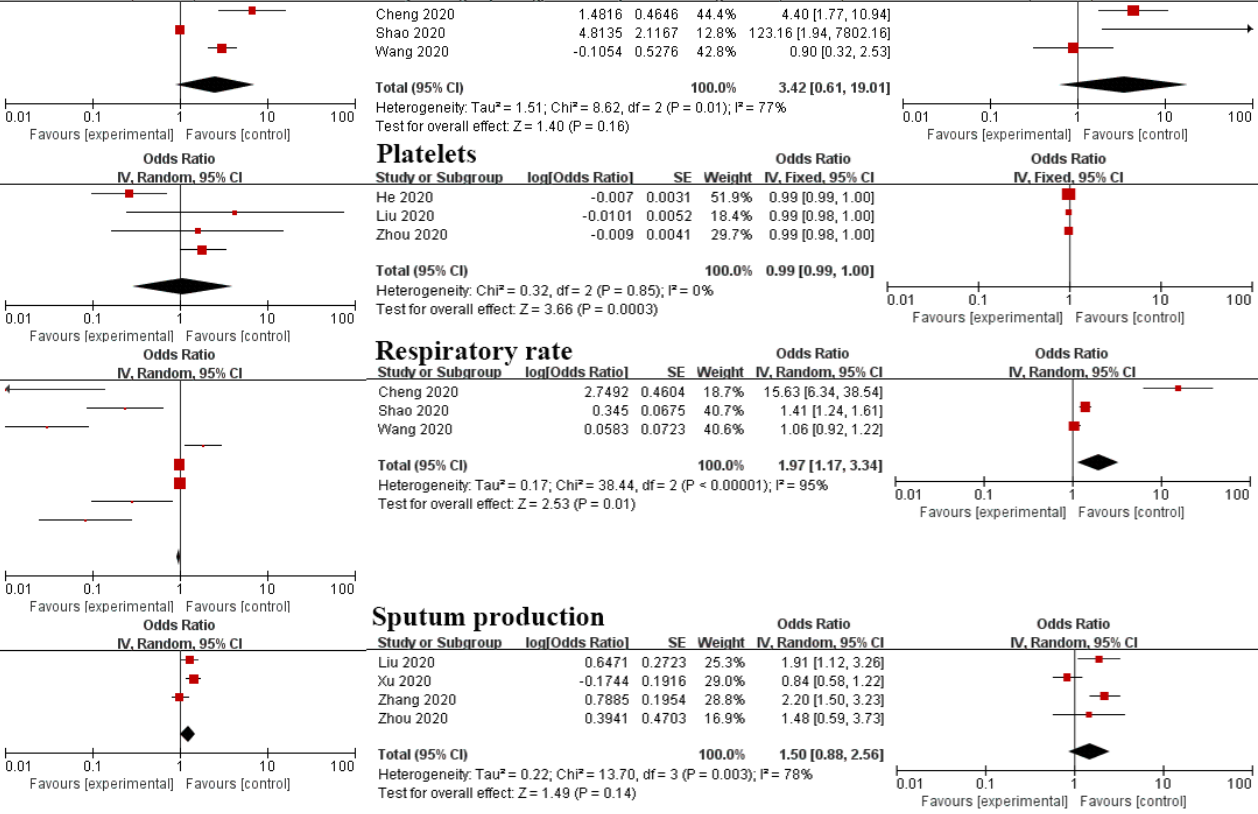

Figure 6. Forest plot of laboratory factors. 


\section{Discussion}

To our knowledge, this is the most up-to-date and complete epidemiological and clinical assessment of determinants for the severity of COVID-19.

This systematic review found that $17.84 \%$ of all cases in China were reported to be severe, and $4.9 \%$ were critical. A study conducted in Europe from 22 March to 3 June 2020 reported $10.4 \%$ (269/2579 cases) severe-to-critical patients [53]. A total of 41 patients (4.9\%) experienced severe or critical diseases in the Silicon Valley region, USA [54]. Apart from that, COVID-19 severity was found to be 51.6\% in Egypt [55]. South Korea reported $11.96 \%$ of cases as severe [56]. It seems that the severity of COVID-19 is related to the economic-development level of countries.

In this review, individuals over 65 years tended to require intensive medical care, and men were more susceptible to severe disease than women were. The same results were also reported by other studies [57-62]. The age difference could be explained by immunological factors; young children are more adept at fighting off novel diseases, whereas the older population is more accustomed to having immune memory responses acquired over a lifetime [63]. Differences in the immune system could also explain why severe COVID-19 is much more common in men than in women $[64,65]$. A history of smoking was not related to the severity of COVID-19, and the same results were also found in a study conducted with a total of 7162 patients, suggesting that smoking is not significantly associated with the severity and mortality of COVID-19. The reasons behind the discrepancy may be linked to the lack of high-quality data and studies about the relationship between smoking and the severity of COVID-19 [66], since some researchers found a positive relationship [67-71]. In addition, there are currently no clinical or laboratory results about the influence of smoking on the disease.

In consideration of symptom factors, cough $[31,39,55,72]$, dyspnoea [22,73,74], fatigue [73-75], and fever [30,31,39,72] were the main symptoms of severe COVID-19. Unsurprisingly, the existence of these symptoms could lead to severe illness. Cough and fever were globally regarded as main factors in previous studies [76,77]. The symptoms could be found in children, adolescents, and adults [78] in research on Europe [79], the UK [80], the United States [81], and other countries. Considering dyspnoea, infection with COVID-19 could result in severe outcomes and death from pneumonia with severe symptoms [82-84]. Fatigue could even be found in the post-COVID-19 period $[85,86]$.

With regard to comorbidities, cancer, cardiovascular diseases, chronic obstructive pulmonary disorder (COPD), diabetes, hypertension, and any other coexisting diseases were related with severity of COVID-19. In the USA, patients with Type I diabetes have a higher possibility of hospitalisation and greater illness severity [87]. Some research suggested that diabetic patients' cytokine responses may be related to the severity of COVID-19 [87,88]. Meta-analysis found that pre-existing COPD was significantly associated with disease severity [89]. However, there are no clinical or laboratory results to explain the relationship between comorbidities and the outcomes of COVID-19. Some research proposed that a lack of respiratory support may cause this situation [90]. In addition, patients with diarrhoea were more likely to have severe disease; to some extent, faecal-oral transmission may result in an increasingly heavy load of the virus that exacerbates the symptoms of the illness [91]. Aggarwal et al. found that pre-existing cardiovascular diseases were associated with worse outcomes among patients with COVID-19. The pathologies of cardiovascular diseases could lead to critical pathomechanisms [92]. Raymond et al. found that hypertension could affect disease progression [93]. Some researchers believe that hypertension and its associated conditions are associated with the pathogenesis of COVID-19 [93], but some studies found no adequate evidence identifying hypertension as a risk factor [94]. In addition, there are different opinions about the effects of cancer on disease severity. Brar found that patients with COVID-19 and cancer showed similar symptoms and severity levels as those of patients without cancer [95]. Lee reported that the outcome of COVID-19 was much worse in patients with cancer [96]. 
In consideration of laboratory factors, higher D-dimer levels were strongly correlated with more severe COVID-19. Other researchers also found that D-dimer levels were higher in severe patients [97]. Additionally, PCT could be a risk factor to predict the severity of COVID-19 [98]. AST [99-101], creatinine [102-104], fibrinogen [105-107], neutrophils $[108,109]$, and respiratory rate $[14,110]$ were reported to be positively related to the severity of COVID-19. Murat et al. found that lymphocyte and platelet levels were higher in positive COVID-19 patients, while C-reactive protein (CRP) was higher in COVID-19negative patients [111]. Wang also found that lower lymphocyte counts and elevated CRP were risk factors for severity [112]. The severity of the disease was related to the presence of opacity in one or more than one lobe [113]. Decreased albumin could be attributed to the severity of COVID-19, and the same results were found by Zhang [8]. In summary, apart from albumin, CRP, lymphocyte counts, and platelet levels, other laboratory factors examined in this review were not associated with the severity of COVID-19.

\section{Conclusions}

In summary, this paper reported the percentages of severe and critical cases of COVID19 (17.84\% and $4.9 \%$, respectively). Furthermore, men, older people, and populations with coexisting diseases were more susceptible to severe disease than their counterparts were. Additionally, patients with cough, dyspnoea, fatigue, fever, and gastrointestinal symptoms tended to experience severe disease. Considering the laboratory results, albumin, AST, creatinine, D-dimer, fibrinogen, neutrophils, procalcitonin, platelets, and respiratory rate were potential factors that could be used to predict the severity of COVID-19. In the future, healthcare workers could use information about these risk factors to take necessary and effective measures to address patients who are likely to progress to severe and critical illness. Other countries could also learn from China's experience.

To our knowledge, this is the first systematic review on determinants of the severity of COVID-19 in China, and the findings provide comprehensive evidence for epidemiological research into this disease. One strength of this review is that it is based on a large sample size: a total of 15,828 cases diagnosed in 18 provinces in China. Another strength is that 148 risk factors were analysed in this review. Behavioural, symptom, comorbidity, laboratory, radiographic, exposure, and other factors were extracted from the included studies. However, there were several limitations of this study. Heterogeneity was quite common, perhaps due to differences among geographic regions and research sites.

Supplementary Materials: The following are available online at https: / www.mdpi.com/article/ 10.3390/su13095305/s1. Table S1: search strategy; Table S2: extracted determinants; Table S3: extracted determinants; Table S4: quality scores assessing risk of bias using a modified NewcastleOttawa scale.

Author Contributions: Conceptualisation, B.Z., Y.M. and N.Z.; methodology, N.Z. and B.Z.; software, N.Z. and B.Z.; validation, R.H., W.N., and T.X.; formal analysis, N.Z.; investigation, N.Z.; resources, N.Z.; data curation, N.Z.; writing-original-draft preparation, N.Z., R.H., W.N., and T.X.; writing-review and editing, Y.M., R.H., W.N., B.Z. and T.X.; visualisation, N.Z.; supervision, Y.M.; project administration, Y.M.; funding acquisition, Y.M. All authors have read and agreed to the published version of the manuscript.

Funding: This research was funded by the Major Program of National Fund of Philosophy and Social Science of China, grant number 17ZDA079.

Institutional Review Board Statement: Not applicable.

Informed Consent Statement: Not applicable.

Data Availability Statement: Data sharing not applicable. No new data were created or analysed in this study. Data sharing is not applicable to this article.

Acknowledgments: The authors would like to thank the National Fund of Philosophy and Social Science of China for its support. 
Conflicts of Interest: The authors declare no conflict of interest.

\section{References}

1. Xu, Z.; Shi, L.; Wang, Y.; Zhang, J.; Huang, L.; Zhang, C.; Liu, S.; Zhao, P.; Liu, H.; Zhu, L.; et al. Pathological findings of COVID-19 associated with acute respiratory distress syndrome. Lancet Respir. Med. 2020, 8, 420-422. [CrossRef]

2. Dong, E.; Du, H.; Gardner, L. An interactive web-based dashboard to track COVID-19 in real time. Lancet Infect. Dis. 2020, 20, 533-534. [CrossRef]

3. Wu, J.T.; Leung, K.; Bushman, M.; Kishore, N.; Niehus, R.; De Salazar, P.M.; Cowling, B.J.; Lipsitch, M.; Leung, G.M. Estimating clinical severity of COVID-19 from the transmission dynamics in Wuhan, China. Nat. Med. 2020, 26, 506-510. [CrossRef] [PubMed]

4. Ng, O.-T.; Marimuthu, K.; Chia, P.-Y.; Koh, V.; Chiew, C.J.; De Wang, L.; Young, B.E.; Chan, M.; Vasoo, S.; Ling, L.-M.; et al. SARS-CoV-2 Infection among Travelers Returning from Wuhan, China. N. Engl. J. Med. 2020, 382, 1476-1478. [CrossRef]

5. Bi, Q.; Wu, Y.; Mei, S.; Ye, C.; Zou, X.; Zhang, Z.; Liu, X.; Wei, L.; A Truelove, S.; Zhang, T.; et al. Epidemiology and transmission of COVID-19 in 391 cases and 1286 of their close contacts in Shenzhen, China: A retrospective cohort study. Lancet Infect. Dis. 2020, 20, 911-919. [CrossRef]

6. World Health Organization. Clinical Management of Severe Acute Respiratory Infection When Novel Coronavirus (nCoV) Infection Is Suspected; WHO Press: Geneva, Switzerland, 2020.

7. National Health and Health Commission of NPC Interim Guidance for Novel Coronavirus Pneumonia (Trial Implementation of Revised Eigthth Edition). 2020. Available online: http://www.gov.cn/zhengce/zhengceku/2020-08/19/content_5535757.htm (accessed on 27 April 2021).

8. Zhang, J.; Wang, X.; Jia, X.; Li, J.; Hu, K.; Chen, G.; Wei, J.; Gong, Z.; Zhou, C.; Yu, H.; et al. Risk factors for disease severity, unimprovement, and mortality in COVID-19 patients in Wuhan, China. Clin. Microbiol. Infect. 2020, 26, 767-772. [CrossRef] [PubMed]

9. Jin, J.-M.; Bai, P.; He, W.; Wu, F.; Liu, X.-F.; Han, D.-M.; Liu, S.; Yang, J.-K. Gender Differences in Patients with COVID-19: Focus on Severity and Mortality. Front. Public Health 2020, 8, 152. [CrossRef]

10. Verity, R.; Okell, L.C.; Dorigatti, I.; Winskill, P.; Whittaker, C.; Imai, N.; Cuomo-Dannenburg, G.; Thompson, H.; Walker, P.G.T.; $\mathrm{Fu}, \mathrm{H}$.; et al. Estimates of the severity of coronavirus disease 2019: A model-based analysis. Lancet Infect. Dis. 2020, $20,669-677$. [CrossRef]

11. Pan, A.; Liu, L.; Wang, C.; Guo, H.; Hao, X.; Wang, Q.; Huang, J.; He, N.; Yu, H.; Lin, X.; et al. Association of Public Health Interventions with the Epidemiology of the COVID-19 Outbreak in Wuhan, China. JAMA 2020, 323, 1915. [CrossRef]

12. Sun, Y.; Dong, Y.; Wang, L.; Xie, H.; Li, B.; Chang, C.; Wang, F.-S. Characteristics and prognostic factors of disease severity in patients with COVID-19: The Beijing experience. J. Autoimmun. 2020, 112, 102473. [CrossRef] [PubMed]

13. Mo, P.; Xing, Y.; Xiao, Y.; Deng, L.; Zhao, Q.; Wang, H.; Xiong, Y.; Cheng, Z.; Gao, S.; Liang, K.; et al. Clinical characteristics of refractory COVID-19 pneumonia in Wuhan, China. Clin. Infect. Dis. 2020. [CrossRef] [PubMed]

14. Robilotti, E.V.; Babady, N.E.; Mead, P.A.; Rolling, T.; Perez-Johnston, R.; Bernardes, M.; Bogler, Y.; Caldararo, M.; Figueroa, C.J.; Glickman, M.S.; et al. Determinants of COVID-19 disease severity in patients with cancer. Nat. Med. 2020, 26, 1218-1223. [CrossRef]

15. Bornstein, S.R.; Rubino, F.; Khunti, K.; Mingrone, G.; Hopkins, D.; Birkenfeld, A.L.; Boehm, B.; Amiel, S.; Holt, R.I.; Skyler, J.S.; et al. Practical recommendations for the management of diabetes in patients with COVID-19. Lancet Diabetes Endocrinol. 2020, 8 , 546-550. [CrossRef]

16. Taquet, M.; Luciano, S.; Geddes, J.R.; Harrison, P.J. Bidirectional associations between COVID-19 and psychiatric disorder: Retrospective cohort studies of 62354 COVID-19 cases in the USA. Lancet Psychiatry 2021, 8, 130-140. [CrossRef]

17. Gross, O.; Moerer, O.; Weber, M.; Huber, T.B.; Scheithauer, S. COVID-19-associated nephritis: Early warning for disease severity and complications? Lancet 2020, 395, e87-e88. [CrossRef]

18. Louapre, C.; Collongues, N.; Stankoff, B.; Giannesini, C.; Papeix, C.; Bensa, C.; Deschamps, R.; Créange, A.; Wahab, A.; Pelletier, J.; et al. Clinical Characteristics and Outcomes in Patients with Coronavirus Disease 2019 and Multiple Sclerosis. JAMA Neurol. 2020, 77, 1079. [CrossRef]

19. Zhang, X.; Tan, Y.; Ling, Y.; Lu, G.; Liu, F.; Yi, Z.; Jia, X.; Wu, M.; Shi, B.; Xu, S.; et al. Viral and host factors related to the clinical outcome of COVID-19. Nat. Cell Biol. 2020, 583, 437-440. [CrossRef]

20. Fajnzylber, J.; Regan, J.; Coxen, K.; Corry, H.; Wong, C.; Rosenthal, A.; Worrall, D.; Giguel, F.; Piechocka-Trocha, A.; Atyeo, C.; et al. SARS-CoV-2 viral load is associated with increased disease severity and mortality. Nat. Commun. 2020, 11, 1-9. [CrossRef] [PubMed]

21. Shrock, E.; Fujimura, E.; Kula, T.; Timms, R.T.; Lee, I.-H.; Leng, Y.; Robinson, M.L.; Sie, B.M.; Li, M.Z.; Chen, Y.; et al. Viral epitope profiling of COVID-19 patients reveals cross-reactivity and correlates of severity. Science 2020, 370, eabd4250. [CrossRef]

22. Li, K.; Wu, J.; Wu, F.; Guo, D.; Chen, L.; Fang, Z.; Li, C. The Clinical and Chest CT Features Associated with Severe and Critical COVID-19 Pneumonia. Investig. Radiol. 2020, 55, 327-331. [CrossRef] [PubMed]

23. Wu, J.; Li, W.; Shi, X.; Chen, Z.; Jiang, B.; Liu, J.; Wang, D.; Liu, C.; Meng, Y.; Cui, L.; et al. Early antiviral treatment contributes to alleviate the severity and improve the prognosis of patients with novel coronavirus disease (COVID-19). J. Intern. Med. 2020, 288, 128-138. [CrossRef] 
24. Domingo, J.L.; Rovira, J. Effects of air pollutants on the transmission and severity of respiratory viral infections. Environ. Res. 2020, 187, 109650. [CrossRef]

25. Fattorini, D.; Regoli, F. Role of the chronic air pollution levels in the COVID-19 outbreak risk in Italy. Environ. Pollut. 2020, 264, 114732. [CrossRef]

26. Gualano, M.R.; Lo Moro, G.; Voglino, G.; Bert, F.; Siliquini, R. Effects of COVID-19 Lockdown on Mental Health and Sleep Disturbances in Italy. Int. J. Environ. Res. Public Health 2020, 17, 4779. [CrossRef]

27. Comunian, S.; Dongo, D.; Milani, C.; Palestini, P. Air Pollution and COVID-19: The Role of Particulate Matter in the Spread and Increase of COVID-19's Morbidity and Mortality. Int. J. Environ. Res. Public Health 2020, 17, 4487. [CrossRef] [PubMed]

28. Walker, P.G.; Whittaker, C.; Watson, O.; Baguelin, M.; Ainslie, K.E.C.; Bhatia, S.; Bhatt, S.; Boonyasiri, A.; Boyd, O.; Cattarino, L. Report 12-The Global Impact of COVID-19 and Strategies for Mitigation and Suppression. 2020. Available online: https: / / www.imperial.ac.uk/mrc-global-infectious-disease-analysis/covid-19/report-12-global-impact-covid-19/ (accessed on 27 April 2021).

29. Leung, K.; Wu, J.T.; Liu, D.; Leung, G.M. First-wave COVID-19 transmissibility and severity in China outside Hubei after control measures, and second-wave scenario planning: A modelling impact assessment. Lancet 2020, 395, 1382-1393. [CrossRef]

30. Wang, J.; Li, Z.; Cheng, X.; Hu, H.; Liao, C.; Li, P.; Lu, J.; Chen, Z. Epidemiologic Characteristics, Transmission Chain, and Risk Factors of Severe Infection of COVID-19 in Tianjin, a Representative Municipality City of China. Front. Public Health 2020, 8, 198. [CrossRef]

31. Zhang, S.-Y.; Lian, J.-S.; Hu, J.-H.; Zhang, X.-L.; Lu, Y.-F.; Cai, H.; Gu, J.-Q.; Ye, C.-Y.; Jin, C.-L.; Yu, G.-D.; et al. Clinical characteristics of different subtypes and risk factors for the severity of illness in patients with COVID-19 in Zhejiang, China. Infect. Dis. Poverty 2020, 9, 1-10. [CrossRef] [PubMed]

32. Wells, G.A.; O'Connell, D.; Peterson, J.; Welch, V.; Losos, M.; Tugwell, P. Newcastle-Ottawa Quality Assessment Scale; Ottawa Hospital Research Institute: Ottawa, ON, Canada, 2014.

33. Higgins, J.P.; Thomas, J.; Chandler, J.; Cumpston, M.; Li, T.; Page, M.J.; Welch, V.A. Cochrane Handbook for Systematic Reviews of Interventions; John Wiley \& Sons: Hoboken, NJ, USA, 2019; pp. 13-29.

34. Hu, X.; Hu, C.; Yang, Y.; Chen, J.; Zhong, P.; Wen, Y.; Chen, X. Clinical characteristics and risk factors for severity of COVID-19 outside Wuhan: A double-center retrospective cohort study of 213 cases in Hunan, China. Ther. Adv. Respir. Dis. 2020, 14, 1-15. [CrossRef]

35. Liu, S.; Luo, H.; Wang, Y.; Cuevas, L.E.; Wang, D.; Ju, S.; Yang, Y. Clinical characteristics and risk factors of patients with severe COVID-19 in Jiangsu province, China: A retrospective multicentre cohort study. BMC Infect. Dis. 2020, 20, 1-9. [CrossRef] [PubMed]

36. Zhou, F.; You, C.; Zhang, X.; Qian, K.; Hou, Y.; Gao, Y.; Zhou, X.-H. Epidemiological Characteristics and Factors Associated with Critical Time Intervals of COVID-19 in Eighteen Provinces, China: A Retrospective Study. Int. J. Infect. Dis. 2021, 102, 123-131. [CrossRef] [PubMed]

37. Liu, W.; Tao, Z.-W.; Wang, L.; Yuan, M.-L.; Liu, K.; Zhou, L.; Wei, P.-F.; Deng, Y.; Liu, J.; Liu, H.-G.; et al. Analysis of factors associated with disease outcomes in hospitalized patients with 2019 novel coronavirus disease. Chin. Med. J. 2020, 133, 1032-1038. [CrossRef]

38. Xu, K.; Zhou, M.; Yang, D.; Ling, Y.; Liu, K.; Bai, T.; Cheng, Z.; Li, J. Application of ordinal logistic regression analysis to identify the determinants of illness severity of COVID-19 in China. Epidemiol. Infect. 2020, 148, e146. [CrossRef] [PubMed]

39. Lian, J.; Jin, X.; Hao, S.; Jia, H.; Cai, H.; Zhang, X.; Hu, J.; Zheng, L.; Wang, X.; Zhang, S.; et al. Epidemiological, clinical, and virological characteristics of 465 hospitalized cases of coronavirus disease 2019 (COVID-19) from Zhejiang province in China. Influ. Other Respir. Viruses 2020, 14, 564-574. [CrossRef]

40. Hu, S.; Wang, W.; Wang, Y.; Litvinova, M.; Luo, K.; Ren, L.; Sun, Q.; Chen, X.; Zeng, G.; Li, J.; et al. Infectivity, susceptibility, and risk factors associated with SARS-CoV-2 transmission under intensive contact tracing in Hunan, China. Nat. Commun. 2021, 12, 1. [CrossRef]

41. Huang, B.; Niu, Y.; Zhao, W.; Bao, P.; Li, D. Reduced Sleep in the Week Prior to Diagnosis of COVID-19 is Associated with the Severity of COVID-19. Nat. Sci. Sleep 2020, 12, 999-1007. [CrossRef]

42. Cai, Q.; Chen, F.; Wang, T.; Luo, F.; Liu, X.; Wu, Q.; He, Q.; Wang, Z.; Liu, Y.; Liu, L.; et al. Obesity and COVID-19 Severity in a Designated Hospital in Shenzhen, China. Diabetes Care 2020, 43, 1392-1398. [CrossRef]

43. Zhou, C.; Huang, Z.; Tan, W.; Li, X.; Yin, W.; Xiao, Y.; Tao, Z.; Geng, S.; Hu, Y. Predictive factors of severe coronavirus disease 2019 in previously healthy young adults: A single-center, retrospective study. Respir. Res. 2020, 21, 1-8. [CrossRef] [PubMed]

44. Hu, L.; Chen, S.; Fu, Y.; Gao, Z.; Long, H.; Ren, H.-W.; Zuo, Y.; Wang, J.; Li, H.; Xu, Q.-B.; et al. Risk Factors Associated with Clinical Outcomes in 323 Coronavirus Disease 2019 (COVID-19) Hospitalized Patients in Wuhan, China. Clin. Infect. Dis. 2020, 71, 2089-2098. [CrossRef]

45. Cen, Y.; Chen, X.; Shen, Y.; Zhang, X.-H.; Lei, Y.; Xu, C.; Jiang, W.-R.; Xu, H.-T.; Zhu, J.; Zhang, L.-L.; et al. Risk factors for disease progression in patients with mild to moderate coronavirus disease 2019-A multi-centre observational study. Clin. Microbiol. Infect. 2020, 26, 1242-1247. [CrossRef] [PubMed]

46. He, F.; Luo, Q.; Lei, M.; Fan, L.; Shao, X.; Huang, G.; Zeng, J.; Zhao, Z.; Qin, S.; Yang, Z.; et al. Risk factors for severe cases of COVID-19: A retrospective cohort study. Aging 2020, 12, 15730-15740. [CrossRef] 
47. Wei, Y.-Y.; Wang, R.-R.; Zhang, D.-W.; Tu, Y.-H.; Chen, C.-S.; Ji, S.; Li, C.-X.; Li, X.-Y.; Zhou, M.-X.; Cao, W.-S.; et al. Risk factors for severe COVID-19: Evidence from 167 hospitalized patients in Anhui, China. J. Infect. 2020, 81, e89-e92. [CrossRef] [PubMed]

48. Li, X.; Xu, S.; Yu, M.; Wang, K.; Tao, Y.; Zhou, Y.; Shi, J.; Zhou, M.; Wu, B.; Yang, Z.; et al. Risk factors for severity and mortality in adult COVID-19 inpatients in Wuhan. J. Allergy Clin. Immunol. 2020, 146, 110-118. [CrossRef] [PubMed]

49. Cheng, S.; Wu, D.; Li, J.; Zou, Y.; Wan, Y.; Shen, L.; Zhu, L.; Shi, M.; Hou, L.; Xu, T.; et al. Risk factors for the critical illness in SARS-CoV-2 infection: A multicenter retrospective cohort study. Respir. Res. 2020, 21, 1-12. [CrossRef]

50. Shao, S.; Zhao, Z.; Wang, F.; Chang, D.; Liu, Y.; Liu, S.; Xu, X.; Li, X.; Jiang, C.; Tang, Z. Risk factors associated with disease aggravation among 126 hospitalized patients with COVID-19 in different places in China: A retrospective observational study. Medicine 2020, 99, e22971. [CrossRef]

51. Gao, C.; Zhao, Z.; Li, F.; Liu, J.-L.; Xu, H.; Zeng, Y.; Yang, L.; Chen, J.; Lu, X.; Wang, C.; et al. The Impact of Individual Lifestyle and Status on the Acquisition of COVID-19: A Case-Control Study. PLoS ONE 2020, 15, e0241540. [CrossRef]

52. Wang, F.; Qu, M.; Zhou, X.; Zhao, K.; Lai, C.; Tang, Q.; Xian, W.; Chen, R.; Li, X.; Li, Z.; et al. The timeline and risk factors of clinical progression of COVID-19 in Shenzhen, China. J. Transl. Med. 2020, 18, 1-11. [CrossRef]

53. Lechien, J.R.; Chiesa-Estomba, C.M.; Vaira, L.A.; De Riu, G.; Cammaroto, G.; Chekkoury-Idrissi, Y.; Circiu, M.; Distinguin, L.; Journe, F.; de Terwangne, C.; et al. Epidemiological, otolaryngological, olfactory and gustatory outcomes according to the severity of COVID-19: A study of 2579 patients. Eur. Arch. Oto-Rhino-Laryngol. 2021, 15, 1-9. [CrossRef]

54. Vaughan, L.; Veruttipong, D.; Shaw, J.G.; Levy, N.; Edwards, L.; Winget, M. Relationship of socio-demographics, comorbidities, symptoms and healthcare access with early COVID-19 presentation and disease severity. BMC Infect. Dis. 2021, 21, 1-10. [CrossRef] [PubMed]

55. Azab, S.M.; Zytoon, A.A.; Kasemy, Z.A.A.; Omar, S.F.; Ewida, S.F.; Sakr, K.A.; Ella, T.F.A. Learning from pathophysiological aspects of COVID-19 clinical, laboratory, and high-resolution CT features: A retrospective analysis of 128 cases by disease severity. Emerg. Radiol. 2021, 2021, 1-15. [CrossRef]

56. Huh, K.; Ji, W.; Kang, M.; Hong, J.; Bae, G.H.; Lee, R.; Na, Y.; Jung, J. Association of prescribed medications with the risk of COVID-19 infection and severity among adults in South Korea. Int. J. Infect. Dis. 2020, 104, 7-14. [CrossRef] [PubMed]

57. Huang, C.C.; Deng, F.M.; Kong, M.X.; Ren, Q.; Melamed, J.; Zhou, M. Reevaluating the concept of "dominant/index tumor nodule" in multifocal prostate cancer. Virchows Arch. Int. J. Pathol. 2014, 464, 589-594. [CrossRef]

58. Khalatbari-Soltani, S.; Cumming, R.G.; Delpierre, C.; Kelly-Irving, M. Importance of collecting data on socioeconomic determinants from the early stage of the COVID-19 outbreak onwards. J. Epidemiol. Community Health 2020, 74, 620-623. [CrossRef] [PubMed]

59. Chen, Y.; Klein, S.L.; Garibaldi, B.T.; Li, H.; Wu, C.; Osevala, N.M.; Li, T.; Margolick, J.B.; Pawelec, G.; Leng, S.X. Aging in COVID-19: Vulnerability, immunity and intervention. Ageing Res. Rev. 2021, 65, 101205. [CrossRef]

60. Ikitimur, H.; Uysal, B.B.; Cengiz, M.; Ikitimur, B.; Uysal, H.; Ozcan, E.; Islamoglu, M.S.; Seyhan, S.; Yavuzer, H.; Yavuzer, S. Determining host factors contributing to disease severity in a family cluster of 29 hospitalized SARS-CoV-2 patients: Could genetic factors be relevant in the clinical course of COVID-19? J. Med. Virol. 2021, 93, 357-365. [CrossRef]

61. Cattaneo, C.; Daffini, R.; Pagani, C.; Salvetti, M.; Mancini, V.; Borlenghi, E.; D’Adda, M.; Oberti, M.; Paini, A.; De Ciuceis, C.; et al. Clinical characteristics and risk factors for mortality in hematologic patients affected by COVID-19. Cancer 2020, 126, 5069-5076. [CrossRef] [PubMed]

62. Pijls, B.G.; Jolani, S.; Atherley, A.; Derckx, R.T.; Dijkstra, J.I.R.; Franssen, G.H.L.; Hendriks, S.; Richters, A.; Venemans-Jellema, A.; Zalpuri, S.; et al. Demographic risk factors for COVID-19 infection, severity, ICU admission and death: A meta-analysis of 59 studies. BMJ Open 2021, 11, e044640. [CrossRef]

63. Brodin, P. Immune determinants of COVID-19 disease presentation and severity. Nat. Med. 2021, 27, 28-33. [CrossRef]

64. Takahashi, T.; Ellingson, M.K.; Wong, P.; Israelow, B.; Lucas, C.; Klein, J.; Silva, J.; Mao, T.; Oh, J.E.; Tokuyama, M.; et al. Sex differences in immune responses that underlie COVID-19 disease outcomes. Nature 2020, 588, 315-320. [CrossRef]

65. Bastard, P.; Rosen, L.B.; Zhang, Q.; Michailidis, E.; Hoffmann, H.-H.; Zhang, Y.; Dorgham, K.; Philippot, Q.; Rosain, J.; Béziat, V.; et al. Auto-antibodies against type I IFNs in patients with life-threatening COVID-19. Science 2020, 370, eabd4585. [CrossRef]

66. Farsalinos, K.; Bagos, P.G.; Giannouchos, T.; Niaura, R.; Barbouni, A.; Poulas, K. Smoking prevalence among hospitalized COVID-19 patients and its association with disease severity and mortality: An expanded re-analysis of a recent publication. Harm Reduct. J. 2021, 18, 1-9. [CrossRef]

67. Vardavas, C.I.; Nikitara, K. COVID-19 and smoking: A systematic review of the evidence. Tob. Induc. Dis. 2020, 18, 20. [CrossRef] [PubMed]

68. Alqahtani, J.S.; Oyelade, T.; Aldhahir, A.M.; Alghamdi, S.M.; Almehmadi, M.; Alqahtani, A.S.; Quaderi, S.; Mandal, S.; Hurst, J.R. Prevalence, Severity and Mortality associated with COPD and Smoking in patients with COVID-19: A Rapid Systematic Review and Meta-Analysis. PLoS ONE 2020, 15, e0233147. [CrossRef] [PubMed]

69. Zhao, Q.; Meng, M.; Kumar, R.; Wu, Y.; Huang, J.; Lian, N.; Deng, Y.; Lin, S. The impact of COPD and smoking history on the severity of COVID-19: A systemic review and meta-analysis. J. Med. Virol. 2020, 92, 1915-1921. [CrossRef] [PubMed]

70. Sanchez-Ramirez, D.C.; Mackey, D. Underlying respiratory diseases, specifically COPD, and smoking are associated with severe COVID-19 outcomes: A systematic review and meta-analysis. Respir. Med. 2020, 171, 106096. [CrossRef] [PubMed] 
71. Singh, A.K.; Gillies, C.L.; Singh, R.; Singh, A.; Chudasama, Y.; Coles, B.; Seidu, S.; Zaccardi, F.; Davies, M.J.; Khunti, K. Prevalence of co-morbidities and their association with mortality in patients with COVID-19: A systematic review and meta-analysis. Diabetes Obes. Metab. 2020, 22, 1915-1924. [CrossRef]

72. Qiu, H.; Wu, J.; Hong, L.; Luo, Y.; Song, Q.; Chen, D. Clinical and epidemiological features of 36 children with coronavirus disease 2019 (COVID-19) in Zhejiang, China: An observational cohort study. Lancet Infect. Dis. 2020, 20, 689-696. [CrossRef]

73. Hu, Y.; Sun, J.; Dai, Z.; Deng, H.; Li, X.; Huang, Q.; Wu, Y.; Sun, L.; Xu, Y. Prevalence and severity of corona virus disease 2019 (COVID-19): A systematic review and meta-analysis. J. Clin. Virol. 2020, 127, 104371. [CrossRef]

74. Chen, G.; Wu, D.; Guo, W.; Cao, Y.; Huang, D.; Wang, H.; Wang, T.; Zhang, X.; Chen, H.; Yu, H.; et al. Clinical and immunological features of severe and moderate coronavirus disease 2019. J. Clin. Investig. 2020, 130, 2620-2629. [CrossRef]

75. Tang, X.; Du, R.-H.; Wang, R.; Cao, T.-Z.; Guan, L.-L.; Yang, C.-Q.; Zhu, Q.; Hu, M.; Li, X.-Y.; Li, Y.; et al. Comparison of Hospitalized Patients with ARDS Caused by COVID-19 and H1N1. Chest 2020, 158, 195-205. [CrossRef]

76. Kazeminia, M.; Jalali, R.; Vaisi-Raygani, A.; Khaledi-Paveh, B.; Salari, N.; Mohammadi, M.; Sabbaghchi, M. Fever and cough are two important factors in identifying patients with the COVID-19: A meta-analysis. J. Mil. Med. 2020, 22, 193-202. [CrossRef]

77. Driessche, K.V.; Nestele, J.; Grouwels, J.; Duval, E.L. Exposure to cough aerosols and development of pulmonary COVID-19. J. Breath Res. 2020, 14, 041003. [CrossRef]

78. Viner, R.M.; Ward, J.L.; Hudson, L.D.; Ashe, M.; Patel, S.V.; Hargreaves, D.; Whittaker, E. Systematic review of reviews of symptoms and signs of COVID-19 in children and adolescents. Arch. Dis. Child. 2020, 1-6. [CrossRef]

79. Lechien, J.R.; Chiesa-Estomba, C.M.; Place, S.; Van Laethem, Y.; Cabaraux, P.; Mat, Q.; Huet, K.; Plzak, J.; Horoi, M.; Hans, S.; et al. Clinical and epidemiological characteristics of 1420 European patients with mild-to-moderate coronavirus disease 2019. J. Intern. Med. 2020, 288, 335-344. [CrossRef]

80. Docherty, A.B.; Harrison, E.M.; Green, C.A.; Hardwick, H.; Pius, R.; Norman, L.; Holden, K.A.; Read, J.M.; Dondelinger, F.; Carson, G.; et al. Features of 16,749 hospitalised UK patients with COVID-19 using the ISARIC WHO Clinical Characterisation Protocol. medRxiv 2020. [CrossRef]

81. Aggarwal, S.; Garcia-Telles, N.; Aggarwal, G.; Lavie, C.; Lippi, G.; Henry, B.M. Clinical features, laboratory characteristics, and outcomes of patients hospitalized with coronavirus disease 2019 (COVID-19): Early report from the United States. Diagnosis 2020, 7, 91-96. [CrossRef]

82. Ora, J.; Liguori, C.; Puxeddu, E.; Coppola, A.; Matino, M.; Pierantozzi, M.; Mercuri, N.B.; Rogliani, P. Dyspnea perception and neurological symptoms in non-severe COVID-19 patients. Neurol. Sci. 2020, 41, 2671-2674. [CrossRef]

83. Horowitz, R.I.; Freeman, P.R.; Bruzzese, J. Efficacy of glutathione therapy in relieving dyspnea associated with COVID-19 pneumonia: A report of 2 cases. Respir. Med. Case Rep. 2020, 30, 101063. [CrossRef]

84. Azabou, E.; Bao, G.; Heming, N.; Bounab, R.; Moine, P.; Chevallier, S.; Chevret, S.; Resche-Rigon, M.; Siami, S.; Sharshar, T.; et al. Randomized Controlled Study Evaluating Efficiency of Low Intensity Transcranial Direct Current Stimulation (tDCS) for Dyspnea Relief in Mechanically Ventilated COVID-19 Patients in ICU: The tDCS-DYSP-COVID Protocol. Front. Med. $2020,7,372$. [CrossRef] [PubMed]

85. Wostyn, P. COVID-19 and chronic fatigue syndrome: Is the worst yet to come? Med. Hypotheses 2021, 146, 110469. [CrossRef]

86. El Sayed, S.; Shokry, D.; Gomaa, S.M. Post-COVID-19 fatigue and anhedonia: A cross-sectional study and their correlation to post-recovery period. Neuropsychopharmacol. Rep. 2021, 41, 50-55. [CrossRef]

87. Gregory, J.M.; Slaughter, J.C.; Duffus, S.H.; Smith, T.J.; LeStourgeon, L.M.; Jaser, S.S.; McCoy, A.B.; Luther, J.M.; Giovannetti, E.R.; Boeder, S.; et al. COVID-19 Severity Is Tripled in the Diabetes Community: A Prospective Analysis of the Pandemic's Impact in Type 1 and Type 2 Diabetes. Diabetes Care 2021, 44, 526-532. [CrossRef]

88. Pal, R.; Bhadada, S.K. COVID-19 and diabetes mellitus: An unholy interaction of two pandemics. Diabetes Metab. Syndr. Clin. Res. Rev. 2020, 14, 513-517. [CrossRef]

89. Rabbani, G.; Islam, S.M.S.; Rahman, M.A.; Amin, N.; Marzan, B.; Robin, R.C.; Alif, S.M. Pre-existing COPD is associated with an increased risk of mortality and severity in COVID-19: A rapid systematic review and meta-analysis. Expert Rev. Respir. Med. 2021, 15, 705-716. [CrossRef] [PubMed]

90. Grasselli, G.; Pesenti, A.; Cecconi, M. Critical Care Utilization for the COVID-19 Outbreak in Lombardy, Italy: Early Experience and Forecast during an Emergency Response. JAMA 2020, 323, 1545. [CrossRef] [PubMed]

91. Ghimire, S.; Sharma, S.; Patel, A.; Budhathoki, R.; Chakinala, R.; Khan, H.; Lincoln, M.; Georgeston, M. Diarrhea Is Associated with Increased Severity of Disease in COVID-19: Systemic Review and Metaanalysis. SN Compr. Clin. Med. 2021, 3, 28-35. [CrossRef]

92. Aggarwal, G.; Cheruiyot, I.; Aggarwal, S.; Wong, J.; Lippi, G.; Lavie, C.J.; Henry, B.M.; Sanchis-Gomar, F. Association of Cardiovascular Disease with Coronavirus Disease 2019 (COVID-19) Severity: A Meta-Analysis. Curr. Probl. Cardiol. 2020, 45, 100617. [CrossRef] [PubMed]

93. Pranata, R.; Lim, M.A.; Huang, I.; Raharjo, S.B.; Lukito, A.A. Hypertension is associated with increased mortality and severity of disease in COVID-19 pneumonia: A systematic review, meta-analysis and meta-regression. J. Renin-Angiotensin-Aldosterone Syst. 2020, 21, 1-11. [CrossRef]

94. Li, G.; Li, H.; Lu, J. No adequate evidence indicating hypertension as an independent risk factor for COVID-19 severity. Clin. Res. Cardiol. 2021, 110, 146-147. [CrossRef] 
95. Brar, G.; Pinheiro, L.C.; Shusterman, M.; Swed, B.; Reshetnyak, E.; Soroka, O.; Chen, F.; Yamshon, S.; Vaughn, J.; Martin, P.; et al. COVID-19 Severity and Outcomes in Patients with Cancer: A Matched Cohort Study. J. Clin. Oncol. 2020, 38, 3914-3924. [CrossRef] [PubMed]

96. Lee, L.Y.; Cazier, J.B.; Starkey, T.; Turnbull, C.D.; UK Coronavirus Cancer Monitoring Project Team; Kerr, R.; Middleton, G. COVID-19 mortality in patients with cancer on chemotherapy or other anticancer treatments: A prospective cohort study. Lancet 2020, 395, 1919-1926. [CrossRef]

97. Gungor, B.; Atici, A.; Baycan, O.F.; Alici, G.; Ozturk, F.; Tugrul, S.; Asoglu, R.; Cevik, E.; Sahin, I.; Barman, H.A. Elevated D-dimer levels on admission are associated with severity and increased risk of mortality in COVID-19: A systematic review and meta-analysis. Am. J. Emerg. Med. 2021, 39, 173-179. [CrossRef]

98. Zhao, C.; Bai, Y.; Wang, C.; Zhong, Y.; Lu, N.; Tian, L.; Cai, F.; Jin, R. Risk factors related to the severity of COVID-19 in Wuhan. Int. J. Med. Sci. 2021, 18, 120-127. [CrossRef] [PubMed]

99. Ghweil, A.A.; Hassan, M.H.; Mohamed, A.K.; Mohamed, A.O.; Mohammed, H.M.; Abdelazez, A.A.; Osman, H.A.; Bazeed, S.E.S Characteristics, Outcomes and Indicators of Severity for COVID-19 Among Sample of ESNA Quarantine Hospital's Patients, Egypt: A Retrospective Study. Infect. Drug Resist. 2020, 13, 2375-2383. [CrossRef] [PubMed]

100. Xie, H.; Zhao, J.; Lian, N.; Lin, S.; Xie, Q.; Zhuo, H. Clinical characteristics of non-ICU hospitalized patients with coronavirus disease 2019 and liver injury: A retrospective study. Liver Int. 2020, 40, 1321-1326. [CrossRef]

101. Ou, M.; Zhu, J.; Ji, P.; Li, H.; Zhong, Z.; Li, B.; Pang, J.; Zhang, J.; Zheng, X. Risk factors of severe cases with COVID-19: A meta-analysis. Epidemiol. Infect. 2020, 148, 1-23. [CrossRef]

102. Izcovich, A.; Ragusa, M.A.; Tortosa, F.; Marzio, M.A.L.; Agnoletti, C.; Bengolea, A.; Ceirano, A.; Espinosa, F.; Saavedra, E.; Sanguine, V.; et al. Prognostic factors for severity and mortality in patients infected with COVID-19: A systematic review. PLoS ONE 2020, 15, e0241955. [CrossRef] [PubMed]

103. Li, J.; He, X.; Yuan, Y.; Zhang, W.; Li, X.; Zhang, Y.; Li, S.; Guan, C.; Gao, Z.; Dong, G. Meta-analysis investigating the relationship between clinical features, outcomes, and severity of severe acute respiratory syndrome coronavirus 2 (SARS-CoV-2) pneumonia. Am. J. Infect. Control. 2021, 49, 82-89. [CrossRef] [PubMed]

104. Malik, P.; Patel, U.; Mehta, D.; Patel, N.; Kelkar, R.; Akrmah, M.; Gabrilove, J.L.; Sacks, H. Biomarkers and outcomes of COVID-19 hospitalisations: Systematic review and meta-analysis. BMJ 2020, 1-12. [CrossRef]

105. Hayıroğlu, M.İ.; Çınar, T.; Tekkeşin, A.İ. Fibrinogen and D-dimer variances and anticoagulation recommendations in Covid-19: Current literature review. Rev. Assoc. Med. Bras. 2020, 66, 842-848. [CrossRef] [PubMed]

106. Lagadinou, M.; E Salomou, E.; Zareifopoulos, N.; Marangos, M.; Gogos, C.; Velissaris, D. Prognosis of COVID-19: Changes in laboratory parameters. Infez. Med. 2020, 28, 89-95.

107. Dong, Y.; Zhou, H.; Li, M.; Zhang, Z.; Guo, W.; Yu, T.; Gui, Y.; Wang, Q.; Zhao, L.; Luo, S.; et al. A novel simple scoring model for predicting severity of patients with SARS-CoV-2 infection. Transbound. Emerg. Dis. 2020, 67, 2823-2829. [CrossRef]

108. Shi, H.; Zuo, Y.; Yalavarthi, S.; Gockman, K.; Zuo, M.; Madison, J.A.; Blair, C.; Woodward, W.; Lezak, S.P.; Lugogo, N.L.; et al Neutrophil calprotectin identifies severe pulmonary disease in COVID-19. J. Leukoc. Biol. 2021, 109, 67-72. [CrossRef] [PubMed]

109. Galloway, J.B.; Norton, S.; Barker, R.D.; Brookes, A.; Carey, I.; Clarke, B.D.; Jina, R.; Reid, C.; Russell, M.D.; Sneep, R.; et al. A clinical risk score to identify patients with COVID-19 at high risk of critical care admission or death: An observational cohort study. J. Infect. 2020, 81, 282-288. [CrossRef] [PubMed]

110. Shim, E.; Tariq, A.; Choi, W.; Lee, Y.; Chowell, G. Transmission potential and severity of COVID-19 in South Korea. Int. J. Infect. Dis. 2020, 93, 339-344. [CrossRef]

111. Seyit, M.; Avci, E.; Nar, R.; Senol, H.; Yilmaz, A.; Ozen, M.; Oskay, A.; Aybek, H. Neutrophil to lymphocyte ratio, lymphocyte to monocyte ratio and platelet to lymphocyte ratio to predict the severity of COVID-19. Am. J. Emerg. Med. 2021, 40, 110-114. [CrossRef] [PubMed]

112. Wang, H.; Xing, Y.; Yao, X.; Li, Y.; Huang, J.; Tang, J.; Zhu, S.; Zhang, Y.; Xiao, J. Retrospective Study of Clinical Features of COVID-19 in Inpatients and Their Association with Disease Severity. Med. Sci. Monit. 2020, 27, e927674-1-e927674-9. [CrossRef]

113. Blain, M.; Kassin, M.T.; Varble, N.; Wang, X.; Xu, Z.; Xu, D.; Carrafiello, G.; Vespro, V.; Stellato, E.; Ierardi, A.M.; et al Determination of disease severity in COVID-19 patients using deep learning in chest X-ray images. Diagn. Interv. Radiol. 2021, 27, 20-27. [CrossRef] [PubMed] 